(c) 2005 International Press

Adv. Theor. Math. Phys. 9 (2005) 1007-1047

\title{
A topological gauged sigma-model
}

\author{
J. M. Baptista
}

Department of Applied Mathematics and Theoretical Physics, University of Cambridge, Wilberforce Road, Cambridge CB3 0WA, UK

j.m.baptista@damtp.cam.ac.uk

\begin{abstract}
We describe a topological field theory that studies the moduli space of solutions of the symplectic vortex equations. It contains as special cases the topological sigma-model and topological Yang-Mills over Kähler surfaces. The correlation functions of the theory are closely related to the recently introduced Hamiltonian Gromov-Witten invariants.
\end{abstract}

\section{Contents}

1. Introduction 1007

$\begin{array}{ll}2 . & \text { The vortex equations } \\ & 1010\end{array}$

3. The manifold of fields 1013

4. The topological action 1018

5. Observables I - definition 1022

e-print archive: http://lanl.arXiv.org/abs/hep-th/0502152 
6. Observables II — "universal" construction

1027

7. Invariants and localization

1034

Acknowledgments

1041

Appendix A: The localization bundle

1042

Appendix B: A proof from Section 7

1044

References

1046

\section{Introduction}

Topological field theories are one of the cornerstones of the modern relations between theoretical physics and mathematics. Their originality stems from the fact that they employ methods of quantum field theory to study problems in geometry; most notably, they use path integrals to obtain invariants of manifolds. The first explicit examples of these theories were topological Yang-Mills and the topological sigma-model, introduced by Witten in $[15,16]$. Topological Yang-Mills studies the moduli space of instantons over a four-manifold. Its correlation functions are then closely related to the Donaldson invariants. The topological sigma-model studies pseudo-holomorphic curves on an almost Kähler manifold, and its correlation functions are essentially the Gromov-Witten invariants. After these initial examples several other topological theories were introduced, for instance 2D topological gravity, Chern-Simons theory and topological string theory, each studying different moduli spaces and invariants. This, however, is very well known story.

The aim of this paper is to define a topological field theory that studies the moduli space of solutions of the symplectic vortex equations. Its correlation functions, as we will see, are then closely related to the so-called Hamiltonian Gromov-Witten invariants. Both the vortex equations and the latter invariants were recently introduced in the mathematics literature by Mundet i Riera in [13], and by Cieliebak et al. in [6]. They have been further studied in $[7,9,14]$. Here we want to give a topological field theory version of the subject.

The setting for the theory is a non-linear gauged sigma-model with Kähler domain $M$ and almost Kähler target $X$. The manifold $X$ should also be equipped with a hamiltonian and holomorphic action of the gauge group $G$. The fields of the theory are then the maps $\phi$ between $M$ and $X$ and the 
$G$-connections $A$ over $M$. The energy functional is defined as

$$
\mathcal{E}(A, \phi)=\int_{M}\left\|F_{A}\right\|^{2}+\left\|\mathrm{d}^{A} \phi\right\|^{2}+\|\mu \circ \phi\|^{2}
$$

where $F_{A}$ is the curvature of $A, \mathrm{~d}^{A} \phi$ is a covariant derivative, and $\mu$ is a moment map for the $G$-action on $X$. Notice that we are not simply gauging the usual sigma-model, since a Maxwell term and a very important potential term are also present. By a Bogomolny argument it can be shown that the energy is minimized by the solutions of

$$
\begin{aligned}
\bar{\partial}^{A} \phi & =0 \\
\Lambda F_{A}+\mu \circ \phi & =0 \\
F_{A}^{0,2} & =0,
\end{aligned}
$$

which are the general vortex equations. All the notation is explained in detail in the next section. Notice that when the group $G$ is trivial, the energy functional and the vortex equations reduce to the usual sigma-model. When the manifold $M$ is four-dimensional and $X$ is a point, we obtain instead the Yang-Mills energy and the equations $\Lambda F_{A}=0$ and $F_{A}^{0,2}=0$, which are just the anti-self-duality equations in disguise. Thus the topological field theory that we want to define will contain as special cases the topological sigma-model (or more precisely the A-model) and topological Yang-Mills over Kähler surfaces. It should also contain the topological gauged linear sigma-model constructed by Witten in [17]; this corresponds to taking $M$ a Riemann surface, $X$ a complex vector space and $G$ a unitary group.

All throughout the paper we approach the topological theory from the point of view of infinite-dimensional differential geometry and equivariant cohomology. This geometrical point of view was pioneered in [1] for topological Yang-Mills and was subsequently applied to many other field theories. It is reviewed for example in $[4,5,8]$. Also, here the manifold $X$ is always assumed to be Kähler, although the formalism could presumably be extended to the almost Kähler case.

We will now give a brief description of the content of each section. Sections 2 and 3 are introductory. In the first one we review the basic facts about the Yang-Mills-Higgs functional and the general vortex equations. In the second one we introduce the geometrical approach to the space of fields of the gauged sigma-model. This consists of an informal presentation of the infinite-dimensional manifold of fields, its tangent space and 1-forms, the action of the group of gauge transformations, and the associated Cartan 
model for equivariant cohomology. Here we try to give a careful exposition and introduce some necessary notation, but all the material is standard.

In Section 4 we contruct the topological Lagrangian for the gauged sigma-model. As is usually the case for cohomological field theories, this Lagrangian can be obtained from purely geometrical considerations. Roughly speaking, it comes directly from the Mathai-Quillen representative of the Thom class of a certain infinite-dimensional vector bundle over the moduli space of fields $[1,4,5,8]$. In Appendix A we explain how the gauged sigma-model can be fitted into this geometrical approach. The results obtained there can then be fed into the standard procedures of $[1,4,5,8]$ in order to justify many of the apparently arbitrary choices in Section 4 . The approach of Section 4, by itself, is a very "nuts and bolts" one, more along the lines of the original constructions in $[15,16]$.

In Section 5 we define the natural observables of the theory. In the geometric picture, these observables are just a set of closed elements of the equivariant complex of the space of fields. Section 6 is then spent explaining in detail the relation between these "quantum" observables and the more traditional ways of defining invariants, namely the so-called universal contructions. All the work of this section (and of most of the paper, by the way) is just a matter of suitably combining and generalizing well known constructions from topological Yang-Mills and the topological sigma-model.

In the first part of Section 7 we apply standard localization to write down the correlation functions as integrals of differential forms over the moduli space of vortex solutions. Using the results of Section 6 , this finally allows us to compare the correlation functions of the topological theory with the Hamiltonian Gromov-Witten invariants defined in [7]. In this reference the invariants have been rigorously defined for $M$ a Riemann surface and a suitable class of $G$-manifolds $X$. The last two subsections are then mostly informative: in Section 7.2 we discuss the moduli space of vortex solutions in the case where $M$ is a Riemann surface; in Section 7.3 we comment on some features of the invariants, namely the wall-crossing phenomena and the adiabatic limit of the vortex equations. Appendix B contains the proof of a proposition stated in Section 7.2 about the vortex moduli space for torus actions.

\section{The vortex equations}

In this section we will go through a quick review of the gauged sigma-model that admits vortex equations. For more details see for example the original references $[6,13]$ or the first section of $[2]$. 
The data we need to define the sigma-model are the following.

- Two Kähler manifolds $M$ and $X$, with respective Kähler forms $\omega_{M}$ and $\omega_{X}$.

- A connected compact Lie group $G$ with Lie algebra $\mathfrak{g}$, and an Adinvariant positive-definite inner product $\kappa(\cdot, \cdot)$ on $\mathfrak{g}$.

- An effective, hamiltonian, left action $\rho$ of $G$ on $X$ such that, for every $g \in G$, the transformations $\rho_{g}: X \rightarrow X$ are holomorphic, and a moment map for this action $\mu: X \rightarrow \mathfrak{g}^{*}$.

- A principal $G$-bundle $\pi_{P}: P \rightarrow M$.

We remark that, in the fullest generality, the complex structure on $X$ need not be assumed integrable, but we will assume that here. Using the elements above one can define the associated bundle $E=P \times_{\rho} X$, which is a bundle over $M$ with typical fiber $X$. It is defined as the quotient of $P \times X$ by the equivalence relation $(p \cdot g, q) \sim(p, g \cdot q)$, for all $g \in G$. The bundle projection $\pi_{E}: E \rightarrow M$ is determined by $\pi_{E}([p, q])=\pi_{P}(p)$, where $[p, q]$ denotes the equivalence class in $E$ of the point $(p, q)$ in $P \times X$.

Definition. The convention used here is that a moment map for the action $\rho$ of $G$ on $\left(X, \omega_{X}\right)$ is a map $\mu: X \rightarrow \mathfrak{g}^{*}$ such that

(i) $\mathrm{d}\langle\mu, \xi\rangle=\iota_{\hat{\xi}} \omega_{X}$ in $\Omega^{1}(X)$ for all $\xi \in \mathfrak{g}$, where $\hat{\xi}$ is the vector field on $X$ defined by the flow $t \mapsto \rho_{\exp (t \xi)}$.

(ii) $\rho_{g}^{*} \mu=\operatorname{Ad}_{g}^{*} \circ \mu$ for all $g \in G$, where $\operatorname{Ad}_{g}^{*}$ is the coadjoint representation of $G$ on $\mathfrak{g}^{*}$.

If a moment map $\mu$ exists, it is not in general unique, but all the other moment maps are of the form $\mu+c$, where $c \in[\mathfrak{g}, \mathfrak{g}]^{0} \subset \mathfrak{g}^{*}$ is a constant in the annihilator of $[\mathfrak{g}, \mathfrak{g}]$. Recall also that under the identification $\mathfrak{g}^{*} \simeq \mathfrak{g}$ provided by an Ad-invariant inner product on $\mathfrak{g}$, the annihilator $[\mathfrak{g}, \mathfrak{g}]^{0}$ is taken to the centre of $\mathfrak{g}$.

The fields of the theory are a connection $A$ on the principal bundle $P$ and a smooth section $\phi$ of $E$. Calling $\mathcal{A}$ the space of such connections and $\Gamma(E)$ the space of such sections, we define the energy functional $\mathcal{E}: \mathcal{A} \times \Gamma(E) \rightarrow \mathbb{R}_{0}^{+}$ of the sigma-model by

$$
\mathcal{E}(A, \phi)=\frac{1}{2} \int_{M} \frac{1}{e^{2}}\left\|F_{A}\right\|^{2}+\left\|\mathrm{d}^{A} \phi\right\|^{2}+e^{2}\|\mu \circ \phi\|^{2}, \quad e \in \mathbb{R}^{+} .
$$

In this formula $F_{A}$ is the curvature of the connection $A$, and $\mathrm{d}^{A} \phi$ is the covariant derivative of $\phi$ induced by $A$. The norms are defined in the natural way, using the metrics on $M, X$ and $\mathfrak{g}$. The last term is well defined because of the $G$-equivariance of the moment map and the $\operatorname{Ad}_{G}$-invariance of the inner product $\kappa$. 
For later convenience we will record here the local (i.e. trivializationdependent) formulae for $\mathrm{d}^{A} \phi$ and $\bar{\partial}^{A} \phi$. Let $s: U \rightarrow P$ be a local section of $P$ over a domain $U$ in $M$. Since $E=P \times_{\rho} X$ is an associated bundle, this determines a trivialization of $\left.E\right|_{U}$ by

$$
U \times\left. X \simeq E\right|_{U}, \quad(x, q) \simeq[s(x), q] .
$$

With respect to these trivializations a section $\phi$ of $E$ can be locally identified with a map $\hat{\phi}: U \rightarrow X$, and a connection $A$ on $P$ can be identified with the connection form $s^{*} A=A_{\mu} \mathrm{d} x^{\mu} \in \Omega^{1}(U ; \mathfrak{g})$. Then the covariant derivative $\mathrm{d}^{A} \phi$, which is a section of the bundle $T^{*} M \otimes \phi^{*} \operatorname{ker} \mathrm{d} \pi_{E} \rightarrow M$, is locally given by

$$
\mathrm{d}^{A} \phi=\mathrm{d} \hat{\phi}+s^{*} A^{a} \hat{e}_{a}=\left(\partial_{\mu} \hat{\phi}^{r}+A_{\mu}^{a} \hat{e}_{a}^{r}\right) \mathrm{d} x^{\mu} \otimes \hat{\phi}^{*}\left(\frac{\partial}{\partial u^{r}}\right) .
$$

In these formulae $\left\{e_{a}\right\}$ is a basis of $\mathfrak{g}, \hat{e}_{a}$ is the vector field on $X$ induced by $e_{a}$ and the left $G$-action, and we have picked real coordinates $\left\{x^{\mu}: 1 \leq \mu \leq 2 m\right\}$ on $M$ and $\left\{u^{r}: 1 \leq r \leq 2 n\right\}$ on $X$. Similarly, by picking complex coordinates $\left\{z^{\alpha}: 1 \leq \alpha \leq m\right\}$ on $M$ and $\left\{w^{j}: 1 \leq j \leq n\right\}$ on $X$, one can also write down the anti-holomorphic part of $\mathrm{d}^{A} \phi$ as

$$
\bar{\partial}^{A} \phi=\bar{\partial} \hat{\phi}+\left(s^{*} A^{a}\right)^{0,1} \hat{e}_{a}=\left(\partial_{\bar{\alpha}} \hat{\phi}^{j}+A_{\bar{\alpha}}^{a} \hat{e}_{a}^{j}\right) \mathrm{d} \bar{z}^{\alpha} \otimes \hat{\phi}^{*}\left(\frac{\partial}{\partial w^{j}}\right) .
$$

Having recorded these formulae we now come to the first basic fact of the theory, namely the existence of a set of first order equations - the vortex equations - whose solutions minimize the energy functional. This was first found in $[6,13]$ for this general non-linear sigma-model.

Theorem. [6,13] For any connection $A \in \mathcal{A}$ and any section $\phi \in \Gamma(E)$,

$$
\mathcal{E}(A, \phi)=T_{[\phi]}+\int_{M}\left\|\bar{\partial}^{A} \phi\right\|^{2}+\frac{1}{2}\left\|\frac{1}{e} \Lambda F_{A}+e \mu \circ \phi\right\|^{2}+\frac{2}{e^{2}}\left\|F_{A}^{0,2}\right\|^{2},
$$

where the term

$$
T_{[\phi]}=\frac{1}{2} \int_{M} \frac{1}{(m-1) !} \phi^{*}\left[\eta_{E}\right] \wedge \omega_{M}^{m-1}-\frac{\kappa_{a b}}{e^{2}(m-2) !} F_{A}^{a} \wedge F_{A}^{b} \wedge \omega_{M}^{m-2}
$$

does not depend on $A$, and only on the homotopy class of $\phi$.

Corollary. [6,13]. Within each homotopy class of the sections $\phi$ we have that $\mathcal{E}(A, \phi) \geq T_{[\phi]}$, and there is an equality if and only if the pair $(A, \phi)$ in $\mathcal{A} \times \Gamma(E)$ satisfies the equations

$$
\begin{aligned}
& \bar{\partial}^{A} \phi=0 \\
& \Lambda F_{A}+e^{2} \mu \circ \phi=0 \\
& F_{A}^{0,2}=0 .
\end{aligned}
$$

These first order equations are usually called vortex equations. 
Besides $\bar{\partial}^{A} \phi$, several new terms appear in (6) when compared with (2); their meaning is the following. The operator $\Lambda: \Omega^{\bullet}(M) \rightarrow \Omega^{\bullet-2}(M)$ is the adjoint, with respect to the metric $g_{M}$ on $M$, of the operator $\eta \mapsto \omega_{M} \wedge \eta$ on $\Omega^{\bullet}(M)$. By well known formulae,

$$
\Lambda F_{A}=*\left(\omega_{M} \wedge * F_{A}\right)=g_{M}\left(F_{A}, \omega_{M}\right),
$$

and so $\Lambda F_{A}$ can be seen as a locally defined function on $M$ with values in $\mathfrak{g}$, just as $\mu \circ \phi$. (More properly, they should be both regarded as global sections of $P \times \operatorname{Ad}_{G} \mathfrak{g}$.) Next, $F_{A}^{0,2}$ is just the $(0,2)$-component of $F_{A}$ under the usual decomposition $\Omega^{2}(M)=\Omega^{2,0} \oplus \Omega^{1,1} \oplus \Omega^{0,2}$. Finally $\left[\eta_{E}\right]$ is a cohomology class in $H^{2}(E)$ that does not depend on $A$. Using the Cartan complex for the $G$-equivariant cohomology of $X,\left[\eta_{E}\right]$ is just the image by the Chern-Weil homomorphism of the cohomology class in $H_{G}^{2}(X)$ determined by the equivariantly closed form $\omega_{X}-\zeta^{b} \mu_{b} \in \Omega_{G}^{2}(X)$ (see for example $[3$, ch. VII]).

\section{The manifold of fields}

\subsection{The manifold}

Here we continue the exposition of the previous section by recalling some well known properties of the infinite-dimensional manifold $\mathcal{A} \times \Gamma(E)$, which is our space of fields. Namely, we describe its natural Kähler structure and the action of the group of gauge transformations $\mathcal{G}$. For more details see for example [13] and the references therein. Along the way we will also write down some explicit expressions that will be needed in Section 4 . In subsections 3.2 and 3.3 the first basic elements of the topological gauged sigma-model are introduced, like the BRST operator $Q$ and a few "anticommuting fields". These are all described in terms of the $\mathcal{G}$-equivariant cohomology of $\mathcal{A} \times \Gamma(E)$.

To start the study of the manifold $\mathcal{A} \times \Gamma(E)$ we first look at its tangent space. Recall that, given a connection $A \in \mathcal{A}$, the tangent space $T_{A} \mathcal{A}$ can be identified with $\Omega^{1}\left(M ; \mathfrak{g}_{P}\right)$ - the space of 1 -forms on $M$ with values in the bundle $\mathfrak{g}_{P}:=P \times \operatorname{Ad}_{G} \mathfrak{g}$. Likewise, given a section $\phi \in \Gamma(E)$, the tangent space $T_{\phi} \Gamma(E)$ can be identified with the space of sections of $\phi^{*}$ Vert $\rightarrow M$. Here Vert $\rightarrow E$ is the sub-bundle of $T E \rightarrow E$ defined by the kernel of $\mathrm{d} \pi_{E}: T E \rightarrow T M$, and $\phi^{*}$ Vert is the pull-back bundle. Thus

$$
T_{(A, \phi)}(\mathcal{A} \times \Gamma(E)) \simeq \Omega^{1}\left(M ; \mathfrak{g}_{P}\right) \oplus \Gamma\left(\phi^{*} \text { Vert }\right) .
$$

Both summands on the right hand side have a natural metric and complex structure, induced by the ones on $M$ and $X$, respectively. Hence the 
manifold $\mathcal{A} \times \Gamma(E)$ has a natural metric and complex structure. Moreover, it can be shown that this complex structure is integrable, compatible with the metric, and that the Kähler form is closed. So $\mathcal{A} \times \Gamma(E)$ is a Kähler manifold.

More explicitly, suppose that we are given tangent vectors

$$
\begin{gathered}
\tau=\tau_{\mu}^{a} \mathrm{~d} x^{\mu} \otimes e_{a} \quad \in \Omega^{1}\left(M ; \mathfrak{g}_{P}\right) \\
V=V^{r} \hat{\phi}^{*}\left(\frac{\partial}{\partial u^{r}}\right) \quad \in \Gamma\left(\phi^{*} \text { Vert }\right)
\end{gathered}
$$

which are here written down in terms of their local representatives with respect to trivializations of $\mathfrak{g}_{P}$ and $E$ induced by a local trivialization of $P$. Then the Kähler metric on $\mathcal{A} \times \Gamma(E)$ is given by

$$
g_{\mathcal{A} \times \Gamma(E)}\left(\tau_{1}+V_{1}, \tau_{2}+V_{2}\right)=\int_{M}\left(\tau_{1}\right)_{\mu}^{a}\left(\tau_{2}\right)_{\nu}^{b}\left(g_{M}\right)^{\mu \nu} \kappa_{a b}+e^{2} V_{1}^{r} V_{2}^{s}\left(g_{X}\right)_{r s}
$$

We now turn to the action of gauge transformations on the fields in $\mathcal{A} \times$ $\Gamma(E)$. Recall that the group $\mathcal{G}$ of gauge transformations is the group of $G$-equivariant automorphisms of the bundle $P \rightarrow M$ which descend to the identity map on the base $M$. Equivalently, $\mathcal{G}$ is the group of sections of the associated bundle $P \times \operatorname{Ad}_{G} G$. Using the local trivializations (3), each of these sections $g \in \mathcal{G}$ is locally represented by a map from $U \subset M$ to $G$. The Lie algebra of $\mathcal{G}$ is the space $\mathfrak{G}=\Omega^{0}\left(M ; \mathfrak{g}_{P}\right)$ of sections of the bundle $\mathfrak{g}_{P} \rightarrow M$; each of these sections can be locally represented by a map from $U$ to $\mathfrak{g}$.

The group $\mathcal{G}$ has a natural right action on the manifold of fields $\mathcal{A} \times \Gamma(E)$. For any $g \in \mathcal{G}$ this action is determined by the formulae

$$
\begin{aligned}
& A \cdot g=\operatorname{Ad}_{g} \circ A-\pi_{P}^{*}\left(g^{-1} \mathrm{~d} g\right) \\
& \phi(x)=[p, q] \Longrightarrow(\phi \cdot g)(x)=\left[p, \rho_{g_{p}^{-1}}(q)\right]
\end{aligned}
$$

In the last formula, $\rho$ is the left $G$-action on $X$ and $g_{p}$ is the only element of $G$ such that $g \circ \pi_{P}(p)=\left[p, g_{p}\right]$ in $P \times{ }_{\mathrm{Ad}_{G}} G$. For our purposes the most relevant facts about these gauge transformations is that they preserve the Kähler metric on $\mathcal{A} \times \Gamma(E)$, as well as the energy functional $\mathcal{E}(A, \phi)$ and the vortex equations. In particular, if $(A, \phi)$ is a solution of the vortex equations, then so is $(A \cdot g, \phi \cdot g)$ for any $g \in \mathcal{G}$. 
The right action of $\mathcal{G}$ on the manifold $\mathcal{A} \times \Gamma(E)$ induces linear maps from the Lie algebra $\mathfrak{G}$ to the tangent spaces $T_{(A, \phi)}(\mathcal{A} \times \Gamma(E))$. These maps correspond to the infinitesimal gauge transformations and are explicitly given by

$$
\begin{aligned}
& C_{(A, \phi)}: \Omega^{0}\left(M ; \mathfrak{g}_{P}\right) \longrightarrow \Omega^{1}\left(M ; \mathfrak{g}_{P}\right) \oplus \Gamma\left(\phi^{*} \text { Vert }\right) \\
& \varepsilon=\varepsilon^{a} e_{a} \longmapsto\left(D_{A} \varepsilon,-\varepsilon^{a} \hat{\phi}^{*}\left(\hat{e}_{a}\right)\right) .
\end{aligned}
$$

Here $D_{A}$ is the covariant derivative on $\mathfrak{g}_{P} \rightarrow M$ induced by the connection $A$ on $P$, and, as explained before, $\hat{e}_{a}$ is the vector field on $X$ induced by $e_{a} \in \mathfrak{g}$ and the left action of $G$ on $X$. Using the inner products on $\mathfrak{G}$ and on the tangent space to $\mathcal{A} \times \Gamma(E)$, one can also consider the adjoint linear maps $C_{(A, \phi)}^{\dagger}=C_{A}^{\dagger} \oplus C_{\phi}^{\dagger}$. A standard calculation shows that these are given by

$$
\begin{aligned}
C_{A}^{\dagger}: \Omega^{1}\left(M ; \mathfrak{g}_{P}\right) & \longrightarrow \Omega^{0}\left(M ; \mathfrak{g}_{P}\right) \\
\tau & \longmapsto-\left(g_{M}\right)^{\mu \nu}\left(\partial_{\nu} \tau_{\mu}-\Gamma_{\nu \mu}^{\lambda} \tau_{\lambda}+\left[A_{\nu}, \tau_{\mu}\right]\right)
\end{aligned}
$$

and

$$
\begin{aligned}
C_{\phi}^{\dagger}: \Gamma\left(\phi^{*} \text { Vert }\right) & \longrightarrow \Omega^{0}\left(M ; \mathfrak{g}_{P}\right) \\
V & \longmapsto-e^{2} \kappa^{a b}\left(g_{X}\right)_{r s}\left(\hat{e}_{b}\right)^{r} V^{s} e_{a},
\end{aligned}
$$

where the $\Gamma_{\nu \mu}^{\lambda}$ 's are the Christoffel symbols for the Levi-Civita connection on $M$.

Finally, there is a moment map for the right action of $\mathcal{G}$ on the Kähler manifold $\mathcal{A} \times \Gamma(E)$. This is a map $\mu_{\mathcal{A} \times \Gamma(E)}: \mathcal{A} \times \Gamma(E) \rightarrow \mathfrak{G}^{*}$ and can be shown to be

$$
\mu_{\mathcal{A} \times \Gamma(E)}(A, \phi)=-\Lambda F_{A}-e^{2} \mu \circ \phi,
$$

where we are using the inner product on $\mathfrak{G}$ to identify this space with a subspace of $\mathfrak{G}^{*}$. Notice that the second vortex equation $(8 \mathrm{~b})$ is exactly the vanishing condition for this moment map.

\subsection{Basic differential forms}

Here we will informally define some basic "coordinate" functions and differential forms on the infinite-dimensional manifold $\mathcal{A} \times \Gamma(E)$, which is our space of fields. As we will see, the definition of these forms depends on a choice of a local trivialization of the bundle $P \rightarrow M$ and of a point $x$ in the domain of this trivialization. This just means that the forms can be tensored with sections of other appropriate bundles in order to define global sections of a bigger bundle over the space of fields. 
More concretely, one proceeds as follows. Let $s$ be a local trivialization of the bundle $P$ over a domain $U$ in $M$. Then given a connection $A \in \mathcal{A}$ and a section $\phi \in \Gamma(E)$, this trivialization allows us to pick local representatives $s^{*} A=A_{\mu}^{a} \mathrm{~d} x^{\mu} e_{a}$ and $\left(\ldots, \hat{\phi}^{r}, \ldots\right)$ for $A$ and $\phi$; just as in (3) and (4). Now, keeping fixed the trivialization $s$, the point $x \in U$, and the indices $a, \mu$ and $r$, the maps $A \mapsto A_{\mu}^{a}(x)$ and $\phi \mapsto \hat{\phi}^{r}(x)$ are actually smooth functions on $\mathcal{A}$ and on an open set of $\Gamma(E)$, respectively. (This is the open set of sections $\phi$ such that the representative $\hat{\phi}(x)$ has values in the domain of the chart $\left\{u^{r}\right\}$ of $X$.) Using the exterior derivative $\tilde{\mathrm{d}}$ on the manifolds $\mathcal{A}$ and $\Gamma(E)$, we can thus define the 1 -forms

$$
\begin{array}{ll}
\psi_{\mu}^{a}(x)=\tilde{\mathrm{d}}\left[A_{\mu}^{a}(x)\right] & \in \Omega^{1}(\mathcal{A}) \\
\chi^{r}(x)=\tilde{\mathrm{d}}\left[\hat{\phi}^{r}(x)\right] & \in \Omega^{1}(\text { open set of } \Gamma(\mathrm{E})) .
\end{array}
$$

It follows from the definition that, acting on the tangent vectors $\tau \in T_{A} \mathcal{A}$ and $V \in T_{\phi} \Gamma(E)$ of (10), these forms give

$$
\begin{aligned}
\psi_{\mu}^{a}(x)[\tau] & =\tau_{\mu}^{a}(x), \\
\chi^{r}(x)[V] & =V^{r}(x) .
\end{aligned}
$$

These trivialization-dependent forms can be combined to define the fields

$$
\begin{aligned}
& \psi=\psi_{\mu}^{a} \wedge \mathrm{d} x^{\mu} \otimes e_{a} \quad \text { and } \\
& \chi=\chi^{r} \hat{\phi}^{*}\left(\frac{\partial}{\partial u^{r}}\right)
\end{aligned}
$$

which are global sections of the bundles

$$
\begin{aligned}
\Lambda^{2}(\mathcal{A} \times M) \otimes \mathfrak{g}_{P} & \longrightarrow \mathcal{A} \times M \quad \text { and } \\
T^{*} \Gamma(E) \otimes \Upsilon^{*} \text { Vert } & \longrightarrow \Gamma(E) \times M,
\end{aligned}
$$

respectively. Here $\Upsilon^{*}$ Vert is the pull-back of Vert $\rightarrow E$ by the natural evaluation map

$$
\Upsilon: \Gamma(E) \times M \longrightarrow E, \quad(\phi, x) \mapsto \phi(x) .
$$

Having in mind the expressions (17), it is also clear that the operators $C_{A}^{\dagger}$ and $C_{\phi}^{\dagger}$ of (13) and (14) can be written as

$$
\begin{aligned}
& C_{A}^{\dagger}=-\left(g_{M}\right)^{\nu \mu}\left(D_{A} \psi\right)_{\nu \mu}^{a} e_{a} \quad \text { and } \\
& C_{\phi}^{\dagger}=-e^{2} \kappa^{a b}\left(g_{X}\right)_{r s}\left(\hat{e}_{b}\right)^{r} \chi^{s} e_{a},
\end{aligned}
$$

where

$$
\left(D_{A} \psi\right)_{\nu \mu}^{a}=\partial_{\nu} \psi_{\mu}^{a}-\Gamma_{\nu \mu}^{\lambda} \psi_{\lambda}^{a}+\left[A_{\nu}, \psi_{\mu}\right]^{a}
$$




\subsection{The $\mathcal{G}$-equivariant complex}

Using the differential forms defined above, let us now look into the $\mathcal{G}$-equivariant complex of the manifold $\mathcal{A} \times \Gamma(E)$. This will lead to the definition of the BRST operator $Q$.

For the sake of clarity we first recall a finite-dimensional example, for instance the $G$-equivariant complex of $X$ in the Cartan model $[3,10]$. This complex is defined as the space

$$
\Omega_{G}(X):=\left(S^{\bullet}\left(\mathfrak{g}^{*}\right) \otimes \Omega^{\bullet}(X)\right)^{G}
$$

of $G$-invariant elements in the tensor product of the symmetric algebra $S^{\bullet}\left(\mathfrak{g}^{*}\right)$ with the de Rham algebra $\Omega^{\bullet}(X)$. The differential operator acting on this space is defined to be $\mathrm{d}_{\mathcal{C}}=\mathrm{d}-e^{a} \otimes \iota_{-\hat{e}_{a}}$, where $\mathrm{d}$ is the exterior derivative on $X,\left\{e^{a}\right\}$ is the basis of $\mathfrak{g}^{*}$ dual to the basis $\left\{e_{a}\right\}$ of $\mathfrak{g}$, and $-\hat{e}_{a}$ are the vector fields on $X$ induced by $e_{a}$ and the right $G$-action. Notice also that, for any $\alpha \in \Omega^{\bullet}(X)$ and $v \in \mathfrak{g}$,

$$
\left(e^{a} \otimes \iota_{-\hat{e}_{a}} \alpha\right)[v]=\iota_{-v^{a} \hat{e}_{a}} \alpha=\hat{e}_{a} \alpha=\iota_{C(v)} \alpha,
$$

where $C: \mathfrak{g} \rightarrow \Gamma(T X)$ is the linear map induced by the right $G$-action on $X$.

In the case of the infinite-dimensional manifold $\mathcal{A} \times \Gamma(E)$ with right $\mathcal{G}$-action, this picture becomes the following. The $\mathcal{G}$-equivariant complex $\Omega_{\mathcal{G}}^{\bullet}(\mathcal{A} \times \Gamma(E))$ is the space of $\mathcal{G}$-invariant sections of the bundle

$$
S^{\bullet}\left(\mathfrak{G}^{*}\right) \otimes \Lambda^{\bullet}(\mathcal{A} \times \Gamma(E)) \longrightarrow \mathcal{A} \times \Gamma(E),
$$

where the first factor in the tensor product is the trivial bundle over $\mathcal{A} \times$ $\Gamma(E)$ with fibre $S^{\bullet}\left(\mathfrak{G}^{*}\right)$, and the second factor is the exterior bundle of the base. The appropriate analog of the differential $\mathrm{d}_{\mathcal{C}}$, which will be specified below, is then what is usually called the $\operatorname{BRST}$ operator $Q$.

To define more explicitly the operator $Q$ we start by introducing the elements $\varphi^{a}(x) \in \mathfrak{G}^{*}$, which are defined by

$$
\varphi^{a}(x)[v]=v^{a}(x) \quad \text { for any } v=v^{a}(x) e_{a} \in \Omega^{0}\left(M ; \mathfrak{g}_{P}\right) .
$$

These elements depend on the choice of local trivialization but can be combined to define the field

$$
\varphi=\varphi^{a}(x) e_{a}
$$

which is a global section of the bundle

$$
\mathfrak{G}^{*} \otimes \mathfrak{g}_{P} \longrightarrow M
$$

Now, having in mind the definition of $\mathrm{d}_{\mathcal{C}}$, it is clear that the analog $Q$ must act on functions on $\mathcal{A} \times \Gamma(E)$ just like $\tilde{\mathrm{d}}$, the exterior derivative. It must 
also annihilate $\varphi^{a}(x)$, just as $\mathrm{d}_{\mathcal{C}}$ annihilates $e^{a}$. Thus

$$
\begin{aligned}
Q A_{\mu}^{a}(x) & =\psi_{\mu}^{a}(x) ; \quad Q \varphi^{a}(x)=0 ; \\
Q \hat{\phi}^{r}(x) & =\chi^{r}(x) .
\end{aligned}
$$

Furthermore, using expressions (12), (17) and (25), one has that for any $v \in \Omega^{0}\left(M ; \mathfrak{g}_{P}\right)$,

$$
\begin{aligned}
& \left.\iota_{C(v)} \psi_{\mu}^{a}(x)\right|_{A}=\left(D_{A} v\right)_{\mu}^{a}(x)=\left(D_{A} \varphi\right)_{\mu}^{a}(x)[v], \\
& \left.\iota_{C(v)} \chi^{r}(x)\right|_{\phi}=-v^{a}(x) \hat{e}_{a}^{r} \circ \hat{\phi}(x)=-\varphi^{a}(x) \hat{e}_{a}^{r} \circ \hat{\phi}(x)[v] .
\end{aligned}
$$

So it follows from the definition of $\mathrm{d}_{\mathcal{C}},(23)$ and the identity $\tilde{\mathrm{d}}^{2}=0$ that

$$
\begin{aligned}
& Q \psi_{\mu}^{a}(x)=-\left(D_{A} \varphi\right)_{\mu}^{a}(x) ; \\
& Q \chi^{r}(x)=\varphi^{a}(x) \hat{e}_{a}^{r} \circ \hat{\phi}(x) .
\end{aligned}
$$

Just as the Cartan operator $\mathrm{d}_{\mathcal{C}}$, one has that $Q^{2}=0$ when acting on $\mathcal{G}$-invariant sections of the bundle $(24)$. When acting on other sections, such as the $A_{\mu}^{a}(x), Q^{2}$ is just like an infinitesimal gauge transformation parametrized by $\varphi$.

\section{The topological action}

The aim of this section is to write down an expression for the action of the topological gauged sigma-model. The approach is a practical one: we introduce the necessary fields, explain what calculations should be performed, and spell out the final answer in (38) and (39). As explained in Introduction, underlying our calculations there is a more fundamental geometrical picture, which justifies the numerous apparently arbitrary choices made here. For more details on this geometrical picture we refer the reader to Appendix A and the reviews $[5,8]$.

The conventions used here are the following. The greek indices $\mu, \nu, \rho \ldots$ and $\alpha, \beta, \gamma \ldots$ refer to real and complex charts, respectively $\left\{x^{\mu}: 1 \leq \mu \leq\right.$ $2 m\}$ and $\left\{z^{\alpha}: 1 \leq \alpha \leq m\right\}$, on the manifold $M$. The latin indices $r, s, t \ldots$ and $i, j, k \ldots$ refer to real and complex charts, respectively and $\left\{u^{r}: 1 \leq r \leq\right.$ $2 n\}$ and $\left\{w^{j}: 1 \leq j \leq n\right\}$, on the manifold $X$. The relations between the real and complex coordinates are the usual ones

$$
z^{\alpha}=x^{2 \alpha-1}+i x^{2 \alpha} \quad \text { and } \quad w^{j}=u^{2 j-1}+i u^{2 j} .
$$

Just as in the real case of Section 3, the complex charts on $M$ and $X$ induce complex coordinates and forms on $\mathcal{A} \times \Gamma(E)$. These are related to the real 
ones by

$$
\begin{array}{ll}
\hat{\phi}_{\mathbb{C}}^{j}=\hat{\phi}^{2 j-1}+i \hat{\phi}^{2 j} ; \quad\left(A_{\mathbb{C}}\right)_{\alpha}^{a}=\overline{\left(A_{\mathbb{C}}\right)_{\bar{\alpha}}^{a}}=\left(A_{2 \alpha-1}^{a}-i A_{2 \alpha}^{a}\right) / 2 ; \\
\chi_{\mathbb{C}}^{j}=\chi^{2 j-1}+i \chi^{2 j} ; \quad\left(\psi_{\mathbb{C}}\right)_{\alpha}^{a}=\overline{\left(\psi_{\mathbb{C}}\right)_{\bar{\alpha}}^{a}}=\left(\psi_{2 \alpha-1}^{a}-i \psi_{2 \alpha}^{a}\right) / 2 .
\end{array}
$$

In the future we will omit the subscript $\mathbb{C}$ and use the type of indices to distinguish real from complex; as for the charts, $\mu$ and $r$ means real, $\alpha$ and $j$ means complex, etc.

Regarding the Kähler geometry of $M$ and $X$, we always work with the holomorphic tangent bundles not the complexified ones. The hermitian metric $h$ is related to the real metric and the Kähler form by

$$
h=h_{\alpha \bar{\beta}} \mathrm{d} z^{\alpha} \otimes \mathrm{d} \bar{z}^{\beta}=g-i \omega .
$$

The hermitian (Levi-Civita) connection satisfies

$$
\nabla_{\frac{\partial}{\partial z^{\alpha}}} \frac{\partial}{\partial z^{\beta}}=\Gamma_{\alpha \beta}^{\gamma} \frac{\partial}{\partial z^{\gamma}}=h^{\gamma \bar{\delta}}\left(\partial_{\alpha} h_{\beta \bar{\delta}}\right) \frac{\partial}{\partial z^{\gamma}},
$$

and the curvature components are

$$
R_{\alpha \bar{\beta} \delta \bar{\gamma}}=-\partial_{\delta} \partial_{\bar{\gamma}} h_{\alpha \bar{\beta}}+h^{\varepsilon \bar{\eta}}\left(\partial_{\delta} h_{\alpha \bar{\eta}}\right)\left(\partial_{\bar{\gamma}} h_{\varepsilon \bar{\beta}}\right) .
$$

The type of indices used distinguishes whether we are working on $M$ or on $X$.

Having stated the conventions, we will now construct the topological action. Firstly we need to introduce several new fields, the so-called antighosts. These are the fields

$$
b_{\bar{\alpha} \bar{\beta}}^{a}(z), \quad c^{a}(z), \quad d_{\bar{\alpha}}^{j}(z), \quad \lambda^{a}(z),
$$

of respective ghost number $-1,-1,-1$ and -2 , and their partners

$$
B_{\bar{\alpha} \bar{\beta}}^{a}(z), \quad C^{a}(z), \quad D_{\bar{\alpha}}^{j}(z), \quad \eta^{a}(z)
$$

of respective ghost number $0,0,0$ and -1 . The BRST operator $Q$ acts on these fields according to the rules

$$
\begin{aligned}
Q b_{\bar{\alpha} \bar{\beta}}^{a} & =B_{\bar{\alpha} \bar{\beta}}^{a} ; \quad Q B_{\bar{\alpha} \bar{\beta}}^{a}=f_{b c}^{a} \varphi^{b} b_{\bar{\alpha} \bar{\beta}}^{c} ; \\
Q c^{a} & =C^{a} ; \quad Q C^{a}=f_{b c}^{a} \varphi^{b} c^{c} ; \\
Q d_{\bar{\alpha}}^{j} & =D_{\bar{\alpha}}^{j}-\Gamma_{i k}^{j} \chi^{k} d_{\bar{\alpha}}^{i} ; \\
Q D_{\bar{\alpha}}^{j} & =R_{i \bar{k} l \bar{m}} h^{j \bar{k}} \chi^{l} \overline{\chi^{m}} d_{\bar{\alpha}}^{i}-\Gamma_{k l}^{j} D_{\bar{\alpha}}^{k} \chi^{l}+\varphi^{a}\left(\nabla \hat{e}_{a}\right)_{k}^{j} d_{\bar{\alpha}}^{k} ; \\
Q \lambda^{a} & =\eta^{a} ; \quad Q \eta^{a}=f_{b c}^{a} \varphi^{b} \lambda^{c} ;
\end{aligned}
$$

where the $f_{b c}^{a}$ are the structure constants of the Lie algebra $\mathfrak{g}$. A geometric interpretation of the antighost fields and of this $Q$-action is given in 
Appendix A. Here we only remark that the relations

$$
Q b=B, \quad Q c=C, \quad Q d=D-\cdots \quad \text { and } \quad Q \lambda=\eta
$$

should be regarded as defining the fields $B, C$ and $D$. In particular, it is the field $D$ that depends explicitly on the metric of the manifold $X$, not the operator $Q$. In fact, $Q$ is a geometric operator that only depends on $M$ and on the $G$-manifold $X$.

As is usually the case with cohomological field theories, the action for our model will be $Q$-exact, i.e. will be of the form

$$
I=Q \Psi,
$$

where $\Psi$ is the so-called gauge fermion. This gauge fermion can be split into two parts

$$
\Psi=\Psi_{\text {localization }}+\Psi_{\text {projection }},
$$

which play different roles in the geometric interpretation of the action. Moreover, in cohomological field theories there is a fairly standard procedure to construct explicit expressions for the gauge fermions. This is reviewed for example in [8]. Going through that procedure in the case of our gauged sigma-model, one gets at the end

$$
\begin{aligned}
\Psi_{\text {loc }}= & \pm i\left(d, \bar{\partial}^{A} \phi\right) \pm \frac{i}{\sqrt{2} e}\left(c, \Lambda F_{A}+e^{2} \mu \circ \phi\right) \pm \frac{\sqrt{2} i}{e}\left(b, F_{A}^{0,2}\right) \\
& +t(b, B)+t(c, C)+t(d, D) ; \\
\Psi_{\text {proj }}= & -i\left(\lambda, C_{A}^{\dagger}+C_{\phi}^{\dagger}\right)
\end{aligned}
$$

where in the first expression $t$ is an arbitrary positive parameter and there are two possible choices of signs. The pairings $(\cdot, \cdot)$ are the natural inner products on the respective spaces. Explicitly, using complex coordinates,

$$
\begin{aligned}
\left(d, \bar{\partial}^{A} \phi\right) & =2 \Re \mathfrak{e} \int_{M} \overline{d_{\bar{\alpha}}^{j}}\left(\bar{\partial}^{A} \phi\right)_{\bar{\beta}}^{k} h^{\alpha \bar{\beta}} h_{k \bar{j}}, \\
\left(c, \Lambda F_{A}+e^{2} \mu \circ \phi\right) & =\int_{M} c^{a}\left(\Lambda F_{A}^{b}+e^{2} \kappa^{b c} \mu_{c} \circ \phi\right) \kappa_{a b}, \\
\left(b, F_{A}^{0,2}\right) & =2 \Re \mathfrak{e} \int_{M} b_{\bar{\alpha} \bar{\beta}}^{a} \overline{\left(F_{A}\right)_{\bar{\gamma} \bar{\delta}}^{c}} h^{\gamma \bar{\alpha}} h^{\delta \bar{\beta}} \kappa_{a c},
\end{aligned}
$$

and the expressions for $(c, C),(d, D)$ and $(b, B)$ are analogous. Rewritting the operators $C^{\dagger}$ of (21) in complex coordinates, we also have that

$$
\left(\lambda, C_{A}^{\dagger}+C_{\phi}^{\dagger}\right)=\int_{M} \lambda^{a} \kappa_{a b} \Re \mathfrak{e}\left[-4 h^{\alpha \bar{\beta}}\left(D_{A} \psi\right)_{\alpha \bar{\beta}}^{b}-e^{2} \kappa^{b c} h_{j \bar{k}}\left(\hat{e}_{c}\right)^{j} \overline{\chi^{k}}\right],
$$


where

$$
\left(D_{A} \psi\right)_{\alpha \bar{\beta}}^{a}=\partial_{\alpha} \overline{\psi_{\beta}^{a}}+f_{b c}^{a} A_{\alpha}^{b} \overline{\psi_{\beta}^{c}} .
$$

The final step is to go from the gauge fermion to the Lagrangian, and this is just a computational matter. One acts with the operator $Q$ on $\Psi$ and integrates out the auxiliary fields $B, C$ and $D$. A few important intermediary stages in this calculation are the following. The section $\Lambda F_{A}$ can be written in real and complex coordinates as

$$
\Lambda F_{A}^{a}=\frac{1}{2} g^{\mu \sigma} g^{\nu \lambda} \omega_{\mu \nu}\left(F_{A}^{a}\right)_{\sigma \lambda}=2 \Im \mathfrak{m}\left[h^{\alpha \bar{\beta}}\left(F_{A}^{a}\right)_{\alpha \bar{\beta}}\right]
$$

where

$$
F_{A}^{a}=\frac{1}{2}\left(F_{A}^{a}\right)_{\mu \nu} \mathrm{d} x^{\mu} \wedge \mathrm{d} x^{\nu}=\Re \mathfrak{e}\left[\left(F_{A}^{a}\right)_{\alpha \beta} \mathrm{d} z^{\alpha} \wedge \mathrm{d} z^{\beta}+\left(F_{A}^{a}\right)_{\alpha \bar{\beta}} \mathrm{d} z^{\alpha} \wedge \mathrm{d} \bar{z}^{\beta}\right]
$$

So

$$
Q \Lambda F_{A}^{a}=4 \Im \mathfrak{m}\left[h^{\alpha \bar{\beta}}\left(D_{A} \psi\right)_{\alpha \bar{\beta}}^{a}\right] .
$$

Using the definition of moment map,

$$
Q\left(\mu_{c} \circ \phi\right)=\Im \mathfrak{m}\left[h_{j \bar{k}}\left(\hat{e}_{c}\right)^{j} \overline{\chi^{k}}\right]
$$

Furthermore, using the holomorphy of the vector fields $\hat{e}_{a}$ and the properties of the hermitian connection $\nabla$ on $X$, one gets that

$$
Q\left[\left(\bar{\partial}^{A} \phi\right)_{\bar{\alpha}}^{j} h_{j \bar{k}}\right]=\left[\left(\left(\phi^{*} \nabla^{A}\right)^{0,1} \chi\right)_{\bar{\alpha}}^{j}+\psi_{\bar{\alpha}}^{a}\left(\hat{e}_{a}\right)^{j}\right] h_{j \bar{k}}+\left(\bar{\partial}^{A} \phi\right)_{\bar{\alpha}}^{j} h_{j \bar{l}} \overline{\Gamma_{k m}^{l} \chi^{m}}
$$

In this last expression

$$
\left[\left(\phi^{*} \nabla^{A}\right)^{0,1} \chi\right]_{\bar{\alpha}}^{j}=\partial_{\bar{\alpha}} \chi^{j}+A_{\bar{\alpha}}^{a} \chi^{k}\left(\nabla \hat{e}_{a}\right)_{k}^{j}+\Gamma_{k l}^{j}\left(\partial_{\bar{\alpha}} \hat{\phi}^{k}\right) \chi^{l}
$$

is the anti-holomorphic part of the connection $\phi^{*} \nabla^{A}$ on the bundle $\phi^{*}$ Vert $\rightarrow$ $M$ induced by $A$ and the hermitian connection on $X$. All throughout the calculation one should also bear in mind that functions on $X$ such as $h_{j \bar{k}}$ or $\left(\hat{e}_{a}\right)^{j}$ depend implicitly on $\phi$, because they are to be evaluated at the point 
$\hat{\phi}(x)$, with $x \in M$. This implies for example that

$$
Q h_{j \bar{k}}=\left(\partial_{l} h_{j \bar{k}}\right) \chi^{l}+\left(\partial_{\bar{l}} h_{j \bar{k}}\right) \overline{\chi^{l}} .
$$

At the end of the calculation, what we get for the localization part of the action is

$$
\begin{aligned}
I_{\mathrm{loc}}= & \frac{1}{4 t}\left[\left\|\bar{\partial}^{A} \phi\right\|_{M}^{2}+\frac{1}{2 e^{2}}\left\|\Lambda F_{A}+e^{2} \mu \circ \phi\right\|_{M}^{2}+\frac{2}{e^{2}}\left\|F_{A}^{0,2}\right\|_{M}^{2}\right] \\
& \mp i\left(d,\left(\phi^{*} \nabla^{A}\right)^{0,1} \chi+\psi_{\bar{\alpha}}^{a} \mathrm{~d} \bar{z}^{\alpha} \otimes \hat{e}_{a}\right) \mp \frac{\sqrt{2} i}{e}\left(b,\left(D_{A} \psi\right)^{0,2}\right) \\
& \mp \frac{i}{\sqrt{2} e} \int_{M} c^{a} \Im \mathfrak{m}\left[4 \kappa_{a b} h^{\alpha \bar{\beta}}\left(D_{A} \psi\right)_{\alpha \bar{\beta}}^{b}-e^{2} h_{j \bar{k}}\left(\hat{e}_{a}\right)^{j} \overline{\chi^{k}}\right] \\
& -t(b,[\varphi, b])-t(c,[\varphi, c]) \\
& +2 t \int_{M} R_{i \bar{j} k \bar{l}} d_{\bar{\alpha}}^{i} \overline{d_{\bar{\beta}}^{j}} \chi^{k} \overline{\chi^{l}} h^{\beta \bar{\alpha}}+\Re \mathfrak{R}\left[\varphi^{a}\left(\nabla \hat{e}_{a}\right)_{i}^{k} d_{\bar{\alpha}}^{i} \overline{d_{\bar{\beta}}^{j}} h_{k \bar{j}} h^{\beta \bar{\alpha}}\right],
\end{aligned}
$$

where $(\cdot, \cdot)$ are the natural inner products of $(32)$ to $(34)$, and the norms $\|\cdot\|_{M}$ come from these inner products. In the expression above we have already integrated out the fields $B, C$ and $D$. Observe that, up to a factor, the bosonic part of $I_{\text {loc }}$ is equal to the classical energy minus the energy $T_{[\phi]}$ (see expression (6)). This quantity is minimized exactly by the vortex solutions.

The calculations for the projection part of the action give

$$
\begin{aligned}
I_{\text {proj }}= & -i\left(\eta, C_{A}^{\dagger}+C_{\phi}^{\dagger}\right) \\
& +i \int_{M} \lambda^{a} \Re \mathfrak{e}\left[4 \kappa_{a b} h^{\alpha \bar{\beta}}\left(f_{b c}^{a} \psi_{\alpha}^{b} \overline{\psi_{\beta}^{c}}-\left(D_{A} D_{A} \varphi\right)_{\alpha \bar{\beta}}^{b}\right)\right. \\
& \left.+e^{2} h_{j \bar{k}}\left(\hat{e}_{a}\right)^{j} \overline{\left(\hat{e}_{c}\right)^{k}} \varphi^{c}+e^{2} h_{j \bar{k}}\left(\nabla \hat{e}_{a}\right)_{l}^{j} \chi^{l} \overline{\chi^{k}}\right],
\end{aligned}
$$

where the first term is similar to (35) and

$$
\left(D_{A} D_{A} \varphi\right)_{\alpha \bar{\beta}}^{b}=\partial_{\bar{\beta}}\left(D_{A} \varphi\right)_{\alpha}^{b}+f_{c d}^{b} A_{\alpha}^{c} \overline{\left(D_{A} \varphi\right)_{\beta}^{b}} .
$$

\section{Observables I - definition}

\subsection{The homomorphism $\mathcal{O}$}

After having defined field content and Lagrangian of our theory, the next natural step is to find an interesting set of observables whose correlation 
functions we would like to compute. The purpose of this section is then to define one such a set. Observables are by definition $Q$-closed elements of $\Omega_{\mathcal{G}}^{\bullet}(\mathcal{A} \times \Gamma(E))$ - the equivariant complex of the space of fields. In this section, roughly speaking, we will define one observable for each given element of $\Omega_{G}^{\bullet}(X)$ - the equivariant complex of $X$. The construction presented here just combines into a single formalism the constructions of observables given in [15] for topological Yang-Mills and in [16] for sigma-models coupled to gauge fields.

Consider the trivial extension of the $\mathcal{G}$-action on $\mathcal{A} \times \Gamma(E)$ to the product manifold $\mathcal{A} \times \Gamma(E) \times M$, and denote by $\Omega_{\mathcal{G}}^{\bullet}(\mathcal{A} \times \Gamma(E) \times M)$ the associated $\mathcal{G}$-equivariant complex. Recall that, as a vector space, this complex is just the space of $\mathcal{G}$-invariant sections of the bundle

$$
S^{\bullet}\left(\mathfrak{G}^{*}\right) \otimes \Lambda^{\bullet}(\mathcal{A} \times \Gamma(E) \times M) \longrightarrow \mathcal{A} \times \Gamma(E) \times M .
$$

The first step towards defining our set of observables will be to construct a homomorphism of complexes

$$
\mathcal{O}: \Omega_{G}^{\bullet}(X) \longrightarrow \Omega_{\mathcal{G}}^{\bullet}(\mathcal{A} \times \Gamma(E) \times M)
$$

This construction involves the sections $\varphi, \psi$ and $\chi$ defined in (26), (18) and (19), respectively, as well as the new sections

$$
\begin{aligned}
F: \mathcal{A} \times M & \longrightarrow \Lambda^{2}(M) \otimes \mathfrak{g}_{P} \\
(A, x) & \longmapsto\left(F_{A}\right)^{a}(x) e_{a}
\end{aligned}
$$

and

$$
\begin{aligned}
\mathbb{D}: \mathcal{A} \times \Gamma(E) \times M & \longrightarrow \Lambda^{1}(M) \otimes \Upsilon^{*} \text { Vert } \\
(A, \phi, x) & \longmapsto\left(\mathrm{d}^{A} \hat{\phi}\right)^{j}(x) \hat{\phi}^{*}\left(\frac{\partial}{\partial w^{j}}\right) .
\end{aligned}
$$

In these formulae $F_{A}$ is the curvature of the connection $A$, and $\mathrm{d}^{A} \phi$ is the covariant derivative of expression (4). Notice also that both $\chi$ and $\mathbb{D}$ can be regarded as sections of the "bigger" bundle

$$
\Lambda^{\bullet}(\mathcal{A} \times \Gamma(E) \times M) \otimes \Upsilon^{*} \text { Vert } \longrightarrow \mathcal{A} \times \Gamma(E) \times M
$$

while $\varphi, \psi$ and $F$ can be regarded as sections of

$$
S^{\bullet}\left(\mathfrak{G}^{*}\right) \otimes \Lambda^{\bullet}(\mathcal{A} \times \Gamma(E) \times M) \otimes \mathfrak{g}_{P} \longrightarrow \mathcal{A} \times \Gamma(E) \times M .
$$

The homomorphism $\mathcal{O}$ can now be defined as follows. Let $\alpha$ be any element of the complex $\Omega_{G}^{\bullet}(X)=\left[S^{\bullet}\left(\mathfrak{g}^{*}\right) \otimes \Omega^{\bullet}(X)\right]^{G}$. It can be locally written as

$$
\alpha=\frac{1}{k ! l !} \alpha_{a_{1} \cdots a_{k} r_{1} \cdots r_{l}}(u) \zeta^{a_{1}} \cdots \zeta^{a_{k}} \mathrm{~d} u^{r_{1}} \wedge \cdots \wedge \mathrm{d} u^{r_{l}},
$$

where $u \in X$ and the coefficients $\alpha_{a_{1} \cdots a_{k} r_{1} \cdots r_{l}}$ are symmetric on the $a_{j}$ 's and anti-symmetric on the $r_{j}$ 's. Then the section $\mathcal{O}_{\alpha} \in \Omega_{\mathcal{G}}^{\bullet}(\mathcal{A} \times \Gamma(E) \times M)$ is 
defined by the local formula

$\mathcal{O}_{\alpha}(A, \phi, x)=\frac{1}{k ! l !}\left(\alpha_{a_{1} \cdots a_{k} r_{1} \cdots r_{l}} \circ \hat{\phi}\right)\left[\prod_{j=1}^{k}\left(\varphi+\psi+F_{A}\right)^{a_{j}}\right]\left[\prod_{i=1}^{l}\left(\chi+\mathrm{d}^{A} \hat{\phi}\right)^{r_{i}}\right]$,

where, on the right hand side, we have omitted the dependence on $x \in M$.

It is not obvious a priori that the homomorphism $\mathcal{O}$ is globally well defined. This is because the local components $(\varphi+\psi+F)^{a},\left(\chi+\mathrm{d}^{A} \hat{\phi}\right)^{r}$ and $\alpha_{a_{1} \cdots a_{k} r_{1} \cdots r_{l}} \circ \hat{\phi}$ depend on the choice of trivialization of $P$, which determines the trivializations of $\mathfrak{g}_{P}$ and $E$. Furthermore, one should also check the invariance of $\mathcal{O}_{\alpha}$ under the $\mathcal{G}$-action on the bundle (40). We will now sketch how all this is done.

Consider a gauge transformation $g \in \mathcal{G}$. It can be locally represented by maps $\hat{g}: U \rightarrow G$, where $U$ is a domain in $M$. One needs to compute the transformation rules of the components $(\varphi+\psi+F)^{a}$ and $\left(\chi+\mathrm{d}^{A} \hat{\phi}\right)^{r}$ under the action of $g$. Notice as well that, since a local gauge transformation is equivalent to a local change of trivialization of $P$ (determined by the transition function $\hat{g}$ ), these rules coincide with the transformation rules of the various components under change of trivialization of $P$.

Let us start with the fields $\varphi, \psi$ and $F$, which are sections of the bundle (45). The left $\mathcal{G}$-action on this bundle is induced by the coadjoint action on $\mathfrak{G}^{*}$, the pull-back action on $\Omega^{\bullet}(\mathcal{A} \times \Gamma(E) \times M)$, and the usual action on $\mathfrak{g}_{P}$. Using the respective definitions one can compute that, under the action of $g \in \mathcal{G}$, the components of the fields transform as

$$
\begin{aligned}
& \varphi^{a}(x) \rightarrow\left(\operatorname{Ad}_{\hat{g}(x)^{-1}}\right)_{b}^{a} \varphi^{b}(x) ; \quad F^{a}(x) \rightarrow\left(\operatorname{Ad}_{\left.\hat{g}(x)^{-1}\right)_{b}^{a} F^{b}(x) ;}\right. \\
& \psi_{\mu}^{a}(x) \rightarrow\left(\operatorname{Ad}_{\left.\hat{g}(x)^{-1}\right)_{b}^{a} \psi_{\mu}^{b}(x) .}\right.
\end{aligned}
$$

On the other hand, the local sections $e_{a}(x)$ of $\mathfrak{g}_{P}$ transform as

$$
e_{a}(x) \rightarrow\left(\operatorname{Ad}_{\hat{g}(x)}\right)_{a}^{b} e_{b}(x) .
$$

This makes apparent the following two facts. Firstly, regarding $\hat{g}$ as a transition function, the sections $\varphi, \psi$ and $F$ defined by (26), (18) and (42) are well defined, i.e. are trivialization independent. Secondly, regarding $\hat{g}$ as a local gauge transformation, the sections $\varphi, \psi$ and $F$ are $\mathcal{G}$-invariant.

The remaining fields $\chi$ and $\mathbb{D}$ are sections of the bundle $(44)$. The left $\mathcal{G}$ action on this bundle is induced by the pull-back action on $\Omega^{\bullet}(\mathcal{A} \times \Gamma(E) \times$ $M)$ and the push-forward action on $\Upsilon^{*}$ Vert. Using the respective definitions one can compute that, under the action of $g \in \mathcal{G}$, the components of these 
fields tranform as

$$
\begin{aligned}
& \chi^{\tilde{r}}(x) \rightarrow\left(\mathrm{d} \rho_{\hat{g}(x)^{-1}}\right)_{s}^{\tilde{r}} \circ \hat{\phi}(x) \quad \chi^{s}(x) ; \\
& \mathbb{D}^{\tilde{r}}(x) \rightarrow\left(\mathrm{d} \rho_{\hat{g}(x)^{-1}}\right)_{s}^{\tilde{r}} \circ \hat{\phi}(x) \mathbb{D}^{s}(x) ;
\end{aligned}
$$

where the tilde over the index $r$ allows for a possible change of chart on the target $X$. On the other hand the local sections of $\Upsilon^{*}$ Vert transform as

$$
\left(g^{-1} \cdot \phi\right)_{x}^{*}\left(\frac{\partial}{\partial \tilde{u}^{r}}\right) \rightarrow\left(\mathrm{d} \rho_{\hat{g}(x)}\right)_{\tilde{r}}^{s} \circ\left(g^{-1} \cdot \phi\right)(x) \phi^{*}\left(\frac{\partial}{\partial u^{s}}\right) .
$$

As before, this makes apparent that $\chi$ and $\mathbb{D}$ are globally well defined as sections of the bundle (44), and that, moreover, they are $\mathcal{G}$-invariant. Finally, substituting all the transformation rules into expression (47), which defines $\mathcal{O}_{\alpha}$, one can compute that

$$
\left(g \cdot \mathcal{O}_{\alpha}\right)_{(A, \phi, x)}=\left(\mathcal{O}_{\hat{g}(x) \cdot \alpha}\right)_{(A, \phi, x)}=\left(\mathcal{O}_{\alpha}\right)_{(A, \phi, x)} \cdot
$$

Here the notation $\hat{g}(x) \cdot \alpha$ refers to the natural $G$-action on $S^{\bullet}\left(\mathfrak{g}^{*}\right) \otimes \Omega^{\bullet}(X)$, and in the last equality we have used that, by assumption, $\alpha$ is $G$-invariant. As before, this shows at the same time that $\mathcal{O}_{\alpha}$ is well defined and $\mathcal{G}$ invariant.

Up to now we have only established that the map $\mathcal{O}$ of (41) is well defined. Since the claim is that $\mathcal{O}$ is a homomorphism of complexes, we must also show that it intertwines the differential operators.

By definition, the differential operator on the $\mathcal{G}$-equivariant complex of $\mathcal{A} \times \Gamma(E)$ is the operator $Q$, presented in Section 3. Thus for the trivial extension of the $\mathcal{G}$-action to $\mathcal{A} \times \Gamma(E) \times M$, the differential operator on the complex $\Omega_{\mathcal{G}}^{\bullet}(\mathcal{A} \times \Gamma(E) \times M)$ is $\mathrm{d}_{M}+Q$, where $\mathrm{d}_{M}$ denotes the exterior derivative on $M$ regarded as acting on forms over $\mathcal{A} \times \Gamma(E) \times M$. Calling $\mathrm{d}_{\mathcal{C}}$ the usual equivariant differential on $\Omega_{G}^{\bullet}(X)$, our aim is to show that

$$
\mathcal{O}_{\mathrm{d}_{\mathcal{C}} \alpha}=\left(\mathrm{d}_{M}+Q\right) \mathcal{O}_{\alpha}
$$

for all $\alpha$ in $\Omega_{G}^{\bullet}(X)$. This implies in particular that $\mathcal{O}$ induces a homomorphism of cohomology groups $H_{G}^{\bullet}(X) \rightarrow H_{\mathcal{G}}^{\bullet}(\mathcal{A} \times \Gamma(E) \times M)$. Having in mind the definition (47) of $\mathcal{O}$, the first step is to see how $\mathrm{d}_{M}+Q$ acts on the fields $\varphi, \psi, F, \chi$ and $\mathbb{D}$. This computation requires the formulae of $(27)$ and (28). After some algebra and several cancelations one gets

$$
\begin{aligned}
\left(\mathrm{d}_{M}+Q\right)\left(\varphi+\psi+F_{A}\right)^{a} & =-\mathrm{d} x^{\mu}\left[A_{\mu}, \varphi+\psi+F_{A}\right]^{a} ; \\
\left(\mathrm{d}_{M}+Q\right)\left(\chi+\mathrm{d}^{A} \hat{\phi}\right)^{r} & =\left(\varphi+\psi+F_{A}\right)^{a}\left(\hat{e}_{a}\right)^{r}-A^{a} \partial_{s}\left(\hat{e}_{a}\right)^{r}\left(\chi+\mathrm{d}^{A} \hat{\phi}\right)^{s} .
\end{aligned}
$$

This computation also uses the identity

$$
A^{a} A^{c}\left(\partial_{s} \hat{e}_{a}^{r}\right) \hat{e}_{c}^{s}=\frac{1}{2}[A, A]^{a} \hat{e}_{a}^{r},
$$


which follows from the usual formula $\left[\hat{e}_{a}, \hat{e}_{c}\right]^{r}=-f_{a c}^{b} \hat{e}_{b}$. Applying these formulae to the definition (47) of $\mathcal{O}_{\alpha}$, a rearrangement of terms shows that

$$
\left(\mathrm{d}_{M}+Q\right) \mathcal{O}_{\alpha}=\mathcal{O}_{\mathrm{d}_{\mathcal{C}} \alpha}+A^{a} \mathcal{O}_{e_{a} \cdot \alpha},
$$

where $e_{a} \cdot \alpha$ refers to the representation of $\mathfrak{g}$ on $S^{\bullet}\left(\mathfrak{g}^{*}\right) \times \Omega^{\bullet}(X)$ induced by the right $G$-action on this space. Since by assumption $\alpha \in \Omega_{G}^{\bullet}(X)$ is $G$-invariant, we have that $e_{a} \cdot \alpha=0$, and so the result follows.

\section{$5.2 \quad$ Natural observables}

Observables of our topological field theory are, by definition, $Q$-closed elements of $\Omega_{\mathcal{G}}^{\bullet}(\mathcal{A} \times \Gamma(E))$. Thus an observable determines a cohomology class in $H_{\mathcal{G}}^{\bullet}(\mathcal{A} \times \Gamma(E))$. Making use of the homomorphism $\mathcal{O}$ defined above, it is now straightforward to construct a large set of observables for our theory. This construction goes just as in References $[15,16]$.

Let $\alpha \in \Omega_{G}^{\bullet}(X)$ be any equivariantly closed form, and consider its image $\mathcal{O}_{\alpha} \in \Omega_{\mathcal{G}}^{\bullet}(\mathcal{A} \times \Gamma(E) \times M)$, which is $\left(\mathrm{d}_{M}+Q\right)$-closed. Decomposing $\mathcal{O}_{\alpha}$ according to the form degree on the $M$ factor, one can write

$$
\mathcal{O}_{\alpha}=\mathcal{O}_{\alpha}^{(0)}+\cdots+\mathcal{O}_{\alpha}^{(2 m)}
$$

where the restriction of $\mathcal{O}_{\alpha}^{(j)}$ to each slice $\left(\varphi^{a}, A, \phi\right) \times M$ is a $j$-form. Moreover, decomposing the identity

$$
\left(\mathrm{d}_{M}+Q\right) \mathcal{O}_{\alpha}=0
$$

according to the form degree on the $M$ factor, one gets the descent equations

$$
\begin{aligned}
\mathrm{d}_{M} \mathcal{O}_{\alpha}^{2 m} & =0 \\
\mathrm{~d}_{M} \mathcal{O}_{\alpha}^{j} & =-Q \mathcal{O}_{\alpha}^{j+1}, \quad 0 \leq j \leq 2 m-1, \\
0 & =Q \mathcal{O}_{\alpha}^{(0)} .
\end{aligned}
$$

Now let $\gamma$ be any $j$-dimensional homology cycle in $M$, and define

$$
W(\alpha, \gamma):=\int_{\gamma} \mathcal{O}_{\alpha}^{j} \quad \in \quad \Omega_{\mathcal{G}}^{\bullet}(\mathcal{A} \times \Gamma(E)) .
$$

As usual, it follows from the descent equations and Stokes' theorem that $W(\alpha, \gamma)$ is $Q$-closed, so it is an observable. Moreover, the cohomology class of $W(\alpha, \gamma)$ in $H_{\mathcal{G}}^{\bullet}(\mathcal{A} \times \Gamma(E))$ only depends on the classes of $\alpha$ and $\gamma$ in $H_{G}^{\bullet}(X)$ and $H_{j}(M)$, respectively. 


\section{Observables II — "universal" construction}

\subsection{The universal construction}

In the last section we saw how to associate with each equivariantly closed form $\alpha \in \Omega_{G}^{\bullet}(X)$ another closed form $\mathcal{O}_{\alpha} \in \Omega_{\mathcal{G}}^{\bullet}(\mathcal{A} \times \Gamma(E) \times M)$. As we will see later, the form $\mathcal{O}_{\alpha}$ can then be "projected down" to a form in $(\mathcal{A} \times$ $\Gamma(E)) / \mathcal{G} \times M$ by, roughly speaking, multiplying it by $e^{-I_{\text {proj }}}$ and performing a certain path integral. This construction corresponds to the "quantum" way of obtaining the topological invariants.

In this section we will describe an alternative "universal" construction that, also roughly speaking, associates directly with each $\alpha$ a certain differential form on the quotient space $(\mathcal{A} \times \Gamma(E)) / \mathcal{G} \times M$. This construction corresponds to the more traditional geometrical approach to the invariants. We will then spend most of the time establishing a result that will later allow us to relate these two constructions. (This result is formula (51), and if the reader is willing to accept it, the subsections 6.2 and 6.3 can be skipped.)

Besides acting on $\mathcal{A} \times \Gamma(E)$, the group of gauge transformations $\mathcal{G}$ also acts on the principal bundle $P$. This action is effective and commutes with the natural $G$-action on $P$. Thus there is a natural action of the group $\mathcal{G} \times G$ on the product space $\mathcal{A} \times \Gamma(E) \times P$. Now let $\mathcal{V}$ be any $\mathcal{G}$-invariant open subset or submanifold of $\mathcal{A} \times \Gamma(E)$ where $\mathcal{G}$ acts freely. Then the action of $\mathcal{G} \times G$ on $\mathcal{V} \times P$ has no fixed points, and in the commutative diagram

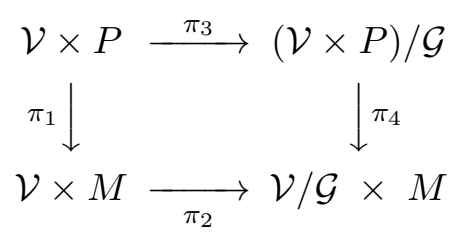

all the quotient maps are principal bundles. More specifically, $\pi_{1}$ and $\pi_{4}$ are $G$-bundles, whereas $\pi_{3}$ and $\pi_{2}$ are $\mathcal{G}$-bundles. We will see later that there are natural connection forms $\theta \in \Omega^{1}(\mathcal{V} \times M ; \mathfrak{G})$ on the bundle $\pi_{2}$ and $\beta \in \Omega^{1}((\mathcal{V} \times P) / \mathcal{G} ; \mathfrak{g})$ on the bundle $\pi_{4}$.

At this point recall the evaluation map $\Upsilon: \Gamma(E) \times M \rightarrow E$ defined in (20). Since elements of $\Gamma(E)$ can be identified with $G$-equivariant maps $P \rightarrow X$, the evaluation map $\Upsilon$ can be identified with a map $\Gamma(E) \times P \rightarrow X$, and this can be trivially extended to

$$
\tilde{\Upsilon}: \mathcal{A} \times \Gamma(E) \times P \longrightarrow X
$$


It follows straightforwardly from the definitions that $\tilde{\Upsilon}$ is $G$-equivariant and is constant on the $\mathcal{G}$-orbits in $\mathcal{A} \times \Gamma(E) \times P$. Thus, restricting to $\mathcal{V}, \tilde{\Upsilon}$ induces a $G$-equivariant map

$$
\hat{\Upsilon}:(\mathcal{V} \times P) / \mathcal{G} \longrightarrow X
$$

Hence given any equivariantly closed form $\alpha \in \Omega_{G}^{\bullet}(X)$, we get by pull-back another equivariantly closed form $\hat{\Upsilon}^{*} \alpha \in \Omega_{G}^{\bullet}((\mathcal{V} \times P) / \mathcal{G})$.

Now it is true on general grounds that the equivariant cohomology of the total space of a principal bundle is isomorphic to the de Rham cohomology of the base space of the bundle. An explicit isomorphism may be constructed by choosing a connection on the bundle and applying the Weil homomorphism $[3,8]$. In our present problem, we can use the connections $\beta$ and $\theta$ to define Weil homomorphisms

$$
\begin{aligned}
& w_{\beta}: \Omega_{\mathcal{G}}^{\bullet}((\mathcal{V} \times P) / \mathcal{G}) \longrightarrow \Omega^{\bullet}((\mathcal{V} \times P) / \mathcal{G})_{G \text {-basic }} \simeq \Omega^{\bullet}(\mathcal{V} / \mathcal{G} \times M) \\
& w_{\theta}: \Omega_{\mathcal{G}}^{\bullet}(\mathcal{V} \times M) \longrightarrow \Omega^{\bullet}(\mathcal{V} \times M)_{\mathcal{G} \text {-basic }} \simeq \Omega^{\bullet}(\mathcal{V} / \mathcal{G} \times M)
\end{aligned}
$$

The aim of this section is to show that

$$
w_{\beta}\left(\hat{\Upsilon}^{*} \alpha\right)=w_{\theta}\left(\mathcal{O}_{\alpha}\right)
$$

as differential forms on the moduli space $\mathcal{V} / \mathcal{G} \times M$. This result is important for the identification of the invariants obtained by quantum field theory methods, with the invariants obtained by more traditional geometrical approaches.

Remark. In Section 7 we will take $\mathcal{V}$ to be the space of solutions of the vortex equations, and it is not always true that $\mathcal{G}$ acts freely on this space. In fact, in the special case of pure Yang-Mills, this never happens, and there one is forced to work with framed connections and deal with the reducible instantons. In the case of our gauged sigma-model one can hope that in some instances this problem will be less acute. This is because a gauge transformation that preserves the connection $A$ in $(A, \phi) \in \mathcal{A} \times \Gamma(E)$ may not preserve the section $\phi$, and so the $\mathcal{G}$-stabilizers will in general be "smaller". This is confirmed in some examples in Section 7.2. This problem nevertheless still requires a more careful study.

\subsection{The natural connections $\theta$ and $\beta$}

The purpose of this subsection is to describe the natural connection forms $\theta$ and $\beta$ mentioned in the discussion above. We will also give some formulae for the curvature forms of these connections. The presentation is rather summarized, and most of the calculations are omitted. 
We will start with the connection $\theta$. As described in Section 3, the right $\mathcal{G}$-action on $\mathcal{V}$ induces operators

$$
C_{(A, \phi)}=C_{A}+C_{\phi}: \mathfrak{G} \longrightarrow T_{(A, \phi)} \mathcal{V}
$$

Using the $\mathcal{G}$-invariant metrics on $\mathcal{V}$ and $\mathfrak{G}$, one then defines the adjoints

$$
C_{(A, \phi)}^{\dagger}=C_{A}^{\dagger}+C_{\phi}^{\dagger}: T_{(A, \phi)} \mathcal{V} \longrightarrow \mathfrak{G}
$$

and $C^{\dagger}$ can be regarded as a 1 -form on $\mathcal{V}$ with values in $\mathfrak{G}$. Since the action of $\mathcal{G}$ on $\mathcal{V}$ is free, the maps $C_{(A, \phi)}$ are injective. Moreover, since the kernel of $C_{(A, \phi)}^{\dagger}$ is the orthogonal complement to the image of $C_{(A, \phi)}$, the linear map

$$
C_{(A, \phi)}^{\dagger} C_{(A, \phi)}: \mathfrak{G} \longrightarrow C_{(A, \phi)}^{\dagger}\left(T_{(A, \phi)} \mathcal{V}\right)
$$

is an isomorphism. One can therefore define a $\mathfrak{G}$-valued form $\theta$ on $\mathcal{V}$ by the formula

$$
\theta_{(A, \phi)}=\left(C_{(A, \phi)}^{\dagger} C_{(A, \phi)}\right)^{-1} \circ C_{(A, \phi)}^{\dagger} .
$$

The $\mathcal{G}$-equivariance of this form follows from the $\mathcal{G}$-invariance of the metrics on $\mathcal{V}$ and $\mathfrak{G}$. Since it is also clear that $\theta_{(A, \phi)} \circ C_{(A, \phi)}=\operatorname{id}_{\mathfrak{G}}$, one concludes that $\theta$ is a connection form for the bundle $\mathcal{V} \rightarrow \mathcal{V} / \mathcal{G}$. This form can be trivially extended to a $\mathfrak{G}$-valued form on the product $\mathcal{V} \times M$, which we also call $\theta$. This extension is a connection form for the bundle $\pi_{2}: \mathcal{V} \times M \rightarrow$ $\mathcal{V} / \mathcal{G} \times M$.

Now we denote by $\mathcal{H}_{\theta}$ and $\mathcal{F}$, respectively, the horizontal distribution and the curvature form on $\mathcal{V}$ determined by the connection $\theta$. It is clear from the definition of $\theta$ that $\mathcal{H}_{\theta}$ is just the orthogonal complement in $T \mathcal{V}$ to the image of $C_{(A, \phi)}$. As for the curvature $\mathcal{F}$, which is a $\mathfrak{G}$-valued 2-form on $\mathcal{V}$, one can compute that

$$
\begin{aligned}
\mathcal{F}_{(A, \phi)}\left(a_{1}+V_{1}, a_{2}+V_{2}\right)= & (\tilde{\mathrm{d}} \theta)_{(A, \phi)}\left(a_{1}+V_{1}, a_{2}+V_{2}\right) \\
= & -2\left(C^{\dagger} C\right)^{-1}\left\{\left[e^{2} \kappa^{a b}\left(g_{X}\right)_{t s} V_{1}^{r} V_{2}^{s}\left(\nabla \hat{e}_{b}\right)_{r}^{t}\right.\right. \\
& \left.\left.+\left(g_{M}\right)^{\mu \nu}\left(a_{1}\right)_{\nu}^{c}\left(a_{2}\right)_{\mu}^{d} f_{c d}^{a}\right] e_{a}\right\}
\end{aligned}
$$

for any horizontal vectors $a_{i}+V_{i} \in \mathcal{H}_{\theta} \subset T_{(A, \phi)} \mathcal{V} \subset T_{A} \mathcal{A} \oplus T_{\phi} \Gamma(E)$. The vertical vectors in $T \mathcal{V}$ are of course annihilated by $\mathcal{F}$. 
Having dealt with $\theta$, we now describe the connection $\beta$ on the bundle $\pi_{4}$. For this we start by recalling the injective linear map

$$
I: \Omega^{k}\left(M ; \mathfrak{g}_{P}\right) \longrightarrow \Omega^{k}(P ; \mathfrak{g})
$$

determined by the formula

$$
\left(\pi_{P}^{*} \nu\right)_{p}\left(Y_{1}, \ldots, Y_{k}\right)=\left[p, I(\nu)\left(Y_{1}, \ldots, Y_{k}\right)\right] \quad \in\left(\mathfrak{g}_{P}\right)_{\pi_{P}(p)} .
$$

Here $\nu$ is any form in $\Omega^{k}\left(M ; \mathfrak{g}_{P}\right), p$ is any point in $P$, and $Y_{j}$ is any vector in $T_{p} P$. The image of this map is exactly the set of $G$-equivariant horizontal forms in $\Omega^{k}(P ; \mathfrak{g})$. In other words, it is the set of forms $\alpha \in \Omega^{k}(P ; \mathfrak{g})$ that satisfy $R_{h}^{*} \alpha=\operatorname{Ad}_{h} \circ \alpha$ and $\iota_{Y} \alpha=0$ for all $h$ in $G$ and all $Y$ in $\operatorname{ker}\left(\mathrm{d} \pi_{P}\right)$. The map $I$ and the connection form $\theta \in \Omega^{1}(\mathcal{V} ; \mathfrak{G})$ allow us to define a form $I \circ \theta \in \Omega^{1}(\mathcal{V} \times P ; \mathfrak{g})$ by the formula

$$
(I \circ \theta)_{(A, \phi, p)}(a+V+Y)=\left.I\left[\theta_{(A, \phi)}(a+V)\right]\right|_{p} \quad \in \mathfrak{g},
$$

where $a+V$ is any vector in $T_{(A, \phi)} \mathcal{V} \subset T_{A} \mathcal{A} \oplus T_{\phi} \Gamma(E)$ and $Y$ is any vector in $T_{p} P$.

Besides $I \circ \theta$, there is another natural form in $\Omega^{1}(\mathcal{V} \times P ; \mathfrak{g})$, which is actually a connection form on the bundle $\pi_{1}$. This is the form $\eta$ defined by the formula

$$
\eta_{(A, \phi, p)}(a+V+Y)=A_{p}(Y) \quad \in \mathfrak{g} .
$$

Thus we can form the combination $\eta+I \circ \theta$, which is a $\mathfrak{g}$-valued 1 -form on the manifold $\mathcal{V} \times P$. A more careful study of this form, which we omit here, then shows that $\eta+I \circ \theta$ descends to a form on the quotient $(\mathcal{V} \times P) / \mathcal{G}$, i.e.

$$
\eta+I \circ \theta=\pi_{3}^{*} \beta
$$

for a unique $\beta \in \Omega^{1}((\mathcal{V} \times P) / \mathcal{G} ; \mathfrak{g})$. Moreover, one can also show that this natural form $\beta$ defines a connection on the bundle $\pi_{4}$, as desired.

Now denote by $F_{\beta}$ the curvature of the connection $\beta$, which is an element of $\Omega^{2}((\mathcal{V} \times P) / \mathcal{G} ; \mathfrak{g})$. A computation using (56) shows that

$$
\begin{aligned}
& \left(\pi_{3}^{*} F_{\beta}\right)_{(A, \phi, p)}\left(Y_{1}, Y_{2}\right)=\left(F_{A}\right)_{p}\left(Y_{1}, Y_{2}\right) \\
& \left(\pi_{3}^{*} F_{\beta}\right)_{(A, \phi, p)}\left(a_{1}+V_{1}, a_{2}+V_{2}\right)=\left.I\left(\mathcal{F}_{(A, \phi)}\left(a_{1}+V_{1}, a_{2}+V_{2}\right)\right)\right|_{p} \\
& \left(\pi_{3}^{*} F_{\beta}\right)_{(A, \phi, p)}(a+V, Y)=I(a)_{p}[Y]
\end{aligned}
$$

for any horizontal vectors $a_{j}+V_{j} \in \mathcal{H}_{\theta} \subset T_{(A, \phi)} \mathcal{V}$ and $Y_{j} \in \mathcal{H}_{A} \subset T_{p} P$.

What we really need for the next subsection, however, is the pull-back of $\pi_{3}^{*} F_{\beta}$ by any local section of the bundle $\pi_{1}$, and this is what we will now 
compute. Let $s: U \rightarrow P$ be a local section of $P$ over a domain $U \subset M$. It determines a local frame of $\mathfrak{g}_{P}$ by

$$
x \longmapsto e_{b}(x):=\left[s(x), e_{b}\right] \in\left(\mathfrak{g}_{P}\right)_{x}
$$

for all $x \in U$ and $e_{b}$ in a basis of $\mathfrak{g}$. Any form $\nu$ in $\Omega^{k}\left(M ; \mathfrak{g}_{P}\right)$ can then be locally written as $\nu=\nu^{b}(x) e_{b}(x)$, and it follows from the definition of the map $I$ that

$$
\nu^{b}=s^{*} I(\nu)^{b} \text { for all } b=1, \ldots, \operatorname{dim} \mathfrak{g} .
$$

In particular we have that, for any $v \in T_{x} U$ and any $a \in \Omega^{1}\left(M ; \mathfrak{g}_{P}\right)$,

$$
I(a)_{s(x)}\left[(\mathrm{d} s)_{x}(v)\right]=a_{x}^{b}(v) e_{b}(x)=\left(\psi_{\mu}^{b} \wedge \mathrm{d} x^{\mu}\right)_{(A, \phi, x)}(a, v) e_{b}(x),
$$

where in the last term we regard $a$ as an element of $T_{A} \mathcal{A}$. Thus considering (57), (58) and the fact that $F_{A}$ and $\mathcal{F}_{(A, \phi)}$ are horizontal forms, we get that

$$
\begin{aligned}
& \left(s^{*} \pi_{3}^{*} F_{\beta}\right)_{(A, \phi, x)}\left(v_{1}, v_{2}\right)=\left(s^{*} F_{A}\right)_{x}\left(v_{1}, v_{2}\right) \\
& \left(s^{*} \pi_{3}^{*} F_{\beta}\right)_{(A, \phi, x)}\left(a_{1}+V_{1}, a_{2}+V_{2}\right)=\left.\mathcal{F}_{(A, \phi)}\left(a_{1}+V_{1}, a_{2}+V_{2}\right)\right|_{x} \\
& \left(s^{*} \pi_{3}^{*} F_{\beta}\right)_{(A, \phi, x)}(a+V, v)=\left(\psi_{\mu} \wedge \mathrm{d} x^{\mu}\right)_{(A, \phi, x)}^{\mathcal{H}_{\theta} \oplus T M}(a+V, v)
\end{aligned}
$$

for any $a_{j}+V_{j} \in T_{(A, \phi)} \mathcal{V}$ and $v_{j} \in T_{x} M$. In this formula the symbol

$$
\left(\psi_{\mu} \wedge \mathrm{d} x^{\mu}\right)^{\mathcal{H}_{\theta} \oplus T M}
$$

denotes the composition of the form $\psi_{\mu} \wedge \mathrm{d} x^{\mu}$ on $\mathcal{V} \times M$ with the projection of vectors

$$
T(\mathcal{V} \times M)=\operatorname{ker}\left(\mathrm{d} \pi_{2}\right) \oplus \mathcal{H}_{\theta} \oplus T M \longrightarrow \mathcal{H}_{\theta} \oplus T M,
$$

i.e., the horizontal part of $\psi_{\mu} \wedge \mathrm{d} x^{\mu}$ with respect to the connection $\theta$ on the bundle $\pi_{2}$. By an abuse of notation, we have also used the same symbol for the section $s$ and its trivial extension $s: \mathcal{V} \times U \rightarrow \mathcal{V} \times P$. The forms $s^{*} \pi_{3}^{*} F_{\beta} \in \Omega^{2}(\mathcal{V} \times U ; \mathfrak{g})$ that we have just calculated will be essential in the next subsection to establish the identity (51).

\subsection{Comparing the two constructions}

The aim of this subsection is to justify equality (51). This equality is the fundamental relation between the "quantum" approach of Section 5 and the universal construction of Section 6.1.

Start by considering the Weil homomorphisms (50), and regard the forms $w_{\beta}\left(\hat{\Upsilon}^{*} \alpha\right)$ and $w_{\theta}\left(\mathcal{O}_{\alpha}\right)$ as basic forms on $(\mathcal{V} \times P) / \mathcal{G}$ and $\mathcal{V} \times M$, respectively. 
Since both these forms descend to $\mathcal{V} / \mathcal{G} \times M$, the commutativity of diagram (49) implies that (51) is equivalent to

$$
\pi_{3}^{*} w_{\beta}\left(\hat{\Upsilon}^{*} \alpha\right)=\pi_{1}^{*} w_{\theta}\left(\mathcal{O}_{\alpha}\right)
$$

It is enough to establish this identity locally, so all we have to do is to show that

$$
w_{\theta}\left(\mathcal{O}_{\alpha}\right)=s^{*} \pi_{3}^{*} w_{\beta}\left(\hat{\Upsilon}^{*} \alpha\right)
$$

where $s: \mathcal{V} \times U \rightarrow \mathcal{V} \times P$ is the local trivialization of $\pi_{1}$ described at the end of the last subsection.

Now let the equivariant form $\alpha$ be as in (46), so that

$$
\hat{\Upsilon}^{*} \alpha=\frac{1}{k ! l !}\left(\alpha_{a_{1} \cdots a_{k} r_{1} \cdots r_{l}} \circ \hat{\Upsilon}\right) \zeta^{a_{1}} \cdots \zeta^{a_{k}}\left(\hat{\Upsilon}^{*} \mathrm{~d} u^{r_{1}}\right) \wedge \cdots \wedge\left(\hat{\Upsilon}^{*} \mathrm{~d} u^{r_{l}}\right)
$$

By definition of the Weil homomorphism, this form is taken to

$$
\begin{aligned}
w_{\beta}\left(\hat{\Upsilon}^{*} \alpha\right)= & \frac{1}{k ! l !}\left(\alpha_{a_{1} \cdots a_{k} r_{1} \cdots r_{l}} \circ \hat{\Upsilon}\right) F_{\beta}^{a_{1}} \wedge \cdots \wedge F_{\beta}^{a_{k}} \\
& \wedge\left(\hat{\Upsilon}^{*} \mathrm{~d} u^{r_{1}}\right)_{\mathrm{hor}} \wedge \cdots \wedge\left(\hat{\Upsilon}^{*} \mathrm{~d} u^{r_{l}}\right)_{\mathrm{hor}},
\end{aligned}
$$

which is a $G$-basic form on $(\mathcal{V} \times P) / \mathcal{G}$. Here the subscript "hor" means the horizontal part of the forms with respect to the connection $\beta$.

Now in general, for any form $\nu$ in $\Omega^{1}(X)$, we have that

$$
\left(\hat{\Upsilon}^{*} \nu\right)_{\mathrm{hor}}=\hat{\Upsilon}^{*} \nu-\beta^{a}\left(\hat{\Upsilon}^{*} \nu\right)\left[\check{e}_{a}\right],
$$

where $\check{e}_{a}$ is the vector field on $(\mathcal{V} \times P) / \mathcal{G}$ associated to $e_{a} \in \mathfrak{g}$ by the right action of $G$. But from the $G$-equivariance of the map $\hat{\Upsilon}$ it is also clear that

$$
(\mathrm{d} \hat{\Upsilon})\left(\check{e}_{a}\right)=-\left(\hat{e}_{a}\right)
$$

where $\hat{e}_{a}$, as usual, is the vector field on $X$ associated to $e_{a} \in \mathfrak{g}$ by the left $G$-action on $X$. Hence one obtains that

$$
\left(\hat{\Upsilon}^{*} \nu\right)_{\text {hor }}=\hat{\Upsilon}^{*} \nu+\beta^{a}\left(\iota_{\hat{e}_{a}} \nu\right) \circ \hat{\Upsilon}
$$

and therefore

$$
\pi_{3}^{*}\left(\hat{\Upsilon}^{*} \nu\right)_{\mathrm{hor}}=\tilde{\Upsilon}^{*} \nu+\left(\pi_{3}^{*} \beta^{a}\right)\left(\iota_{\hat{e}_{a}} \nu\right) \circ \tilde{\Upsilon} .
$$

On the other hand, it follows from (56), (55) and (58) that

$$
\left.s^{*} \pi_{3}^{*} \beta^{a}\right|_{(A, \phi, x)}=\left(s^{*} A^{a}\right)_{x}+\theta_{(A, \phi)}^{a} .
$$

Moreover, tautologically,

$$
\tilde{\Upsilon} \circ s(A, \phi, x)=\hat{\phi}(x),
$$


where $\hat{\phi}: U \rightarrow X$ is the local representative of $\phi \in \Gamma(E)$ with respect to the trivialization of $E$ induced by $s$. Thus the pull-back by $s$ of equation (62) is

$$
\left.s^{*} \pi_{3}^{*}\left(\hat{\Upsilon}^{*} \nu\right)_{\text {hor }}\right|_{(A, \phi, x)}=s^{*} \tilde{\Upsilon}^{*} \nu+\left(\iota_{\hat{e}_{a}} \nu\right) \circ \hat{\phi}\left[s^{*} A^{a}+\theta^{a}\right],
$$

where on the right hand side we have omitted the dependence on $x \in M$.

The equation above will now be applied to the particular case where $\nu$ is the local 1-form $\mathrm{d} u^{r}$. Denoting by $\tilde{\mathrm{d}}$ the exterior derivative on $\mathcal{A} \times \Gamma(E) \times$ $M$, and noting that $\tilde{\mathrm{d}}$ coincides with the equivariant differential $\mathrm{d}_{M}+Q$ when acting on functions, it follows from (63) that

$$
\left.s^{*} \tilde{\Upsilon}^{*} \mathrm{~d} u^{r}\right|_{(A, \phi, x)}=\tilde{\mathrm{d}}\left[\hat{\phi}^{r}(x)\right]=\left(\mathrm{d} \hat{\phi}^{r}\right)_{x}+\left.\chi^{r}(x)\right|_{\phi} .
$$

On the other hand, considering the horizontal projection (60), one can compute that the component in $\mathcal{H}_{\theta} \oplus T M$ of the 1 -form $\chi^{r}(x) \in T_{(\mathcal{A}, \phi, x)}^{*}(\mathcal{V} \times M)$ is given by

$$
\begin{aligned}
{\left[\chi^{r}(x)\right]^{\mathcal{H}_{\theta} \oplus T M}(a+V+v) } & =\chi^{r}(x)\left[a+V+v-C_{(A, \phi)} \circ \theta(a+V+v)\right] \\
& =\left[\chi^{r}(x)+\hat{e}_{b}^{r} \circ \hat{\phi}(x) \theta^{b}\right](a+V+v) .
\end{aligned}
$$

Hence it follows from (64) that

$$
\left.s^{*} \pi_{3}^{*}\left(\hat{\Upsilon}^{*} \mathrm{~d} u^{r}\right)_{\text {hor }}\right|_{(A, \phi, x)}=\left(\mathrm{d}^{A} \hat{\phi}^{r}\right)_{x}+\left[\chi^{r}(x)\right]_{(A, \phi, x)}^{\mathcal{H}_{\theta} \oplus T M} .
$$

With the formula above at hand, it is now possible to compute the pull-back by $\pi_{3} \circ s$ of equation (61). In fact, making use of (59) and (65), we have that

$$
\begin{aligned}
\left.s^{*} \pi_{3}^{*} w_{\beta}\left(\hat{\Upsilon}^{*} \alpha\right)\right|_{(A, \phi, x)}= & \frac{1}{k ! l !} \alpha_{a_{1} \cdots a_{k} r_{1} \cdots r_{l}} \circ \hat{\phi}(x) \\
& {\left[\prod_{1 \leq j \leq k}\left(\mathcal{F}^{a_{j}}+\psi_{\mu}^{a_{j}} \wedge \mathrm{d} x^{\mu}+F^{a_{j}}\right)_{(A, \phi, x)}\right]^{\mathcal{H}_{\theta} \oplus T M} } \\
& \wedge\left[\prod_{1 \leq i \leq l}\left(\chi^{r_{i}}+\mathrm{d}^{A} \hat{\phi}^{r_{i}}\right)_{(A, \phi, x)}\right]^{\mathcal{H}_{\theta} \oplus T M}
\end{aligned}
$$

where we have also used that $\mathcal{F}_{(A, \phi)}^{a},\left(F_{A}^{a}\right)_{x}$ and $\left(\mathrm{d}^{A} \hat{\phi}^{r}\right)_{x}$, regarded as forms in $\Lambda_{(A, \phi, x)}^{\bullet}(\mathcal{V} \times M)$, are already horizontal with respect to the projection $T(\mathcal{V} \times M) \rightarrow \mathcal{H}_{\theta} \oplus T M$.

The final step is to compare the expression above with the definition of the equivariant form $\mathcal{O}_{\alpha}$. It is then clear that (66) can be obtained from (47) by substituting $\varphi^{a} \rightarrow \mathcal{F}^{a}$ and taking the $\mathcal{H}_{\theta} \oplus T M$-component of the resulting form. But as is well known, this is precisely the definition of the 
Weil homomorphism

$$
w_{\theta}: \Omega_{\mathcal{G}}^{\bullet}(\mathcal{V} \times M) \longrightarrow \Omega^{\bullet}(\mathcal{V} \times M)_{\mathcal{G} \text {-basic }}
$$

associated with the connection $\theta$ on the bundle $\mathcal{V} \times M \rightarrow \mathcal{V} / \mathcal{G} \times M$. Thus the right hand side of (51) coincides with $w_{\theta}\left(\mathcal{O}_{\alpha}\right)$, as desired.

\section{Invariants and localization}

\subsection{Correlation functions and localization}

The purpose of this final section is to study the correlation functions of the observables $W(\alpha, \gamma)$ defined in Section 5. As is usual in topological field theory, the importance of these correlation functions stems from the fact that they are expected to be invariant under deformations of the metric and complex structure of the manifolds $M$ and $X$. This means that they essentially only depend on the $G$-action and on the differentiable/symplectic structures of $M$ and $X$, and hence are potentially able to distinguish inequivalent manifolds and $G$-actions. Another important property of the correlation functions is that, while they are defined by a certain path-integral over the space of all fields, their computation can be reduced to an integral over the moduli space of solutions of the vortex equations.

We point out that the methods of this first subsection are standard, as the localization arguments that apply to topological Yang-Mills, for instance, can be straightforwardly transposed to our gauged sigma-model, at least at a heuristical level. Thus, besides the original references $[15,16]$, we follow closely the review [8, ch. 14].

The correlation functions of the observables $W(\alpha, \gamma)$ are of the form

$$
Z\left(\ldots, \alpha_{i}, \gamma_{i}, \ldots\right):=\int \mathcal{D}(b, c, d, \varphi, \eta, \lambda, A, \psi, \phi, \chi) e^{-I_{\mathrm{loc}}-I_{\mathrm{proj}}} \prod_{i} W\left(\alpha_{i}, \gamma_{i}\right) .
$$

Since the path-integral measure is assumed not to depend on the metric and complex structure on $M$ and $X$, the dependence of $Z$ on these quantities is contained in

$$
I_{\mathrm{loc}}+I_{\mathrm{proj}}=Q\left(\Psi_{\mathrm{loc}}+\Psi_{\mathrm{proj}}\right) .
$$

Therefore under a small deformation $\delta g_{\mu \nu}$ of the metric on $M$, for example, the change $\delta Z$ is given by the path integral of

$$
e^{-I_{\mathrm{loc}}-I_{\mathrm{proj}}} Q\left[\left(\delta \Psi_{\mathrm{loc}}+\delta \Psi_{\mathrm{proj}}\right) \prod_{i} W\left(\alpha_{i}, \gamma_{i}\right)\right]
$$


where we have used that the $W^{\prime} s$ are $Q$-closed and that the differential $Q$ does not depend on $g_{\mu \nu}$ (see the remarks after (30)). This last path integral represents the vacuum expectation value of a $Q$-exact quantity, and by standard heuristical arguments it vanishes $[15,16]$. One therefore expects the correlation functions $Z$ to be invariant under deformations of the metric and complex structure of $M$ and $X$ (see however Section 7.3).

On the other hand there is the localization argument, which reduces the path integral defining $Z$ to an integral over the moduli space of vortex solutions. In order to state this result, let $\mathcal{V}$ denote the space of solutions of the vortex equations, and assume that $\mathcal{G}$ acts freely on $\mathcal{V}$. The basic localization result, as stated in [8], asserts that

$$
\begin{aligned}
Z & =\int_{(A, \phi) \in \mathcal{V}} \mathcal{D}(A, \phi, \psi, \chi, \varphi, \eta, \lambda)\left(\prod_{i} W_{i}\right) \wedge \mathcal{E}(\operatorname{cok} \mathbb{O} \rightarrow \mathcal{V}) e^{-I_{\text {proj }}} \\
& =\int_{\mathcal{V} / \mathcal{G}}\left[\prod_{i} w_{\theta}\left(W_{i}\right)\right] \wedge \mathcal{E}(\operatorname{cok} \mathbb{O} / \mathcal{G} \rightarrow \mathcal{V} / \mathcal{G}) .
\end{aligned}
$$

We now explain the notation in this formula. $\mathbb{O}$ is the linear operator defined at each $(A, \phi) \in \mathcal{A} \times \Gamma(E)$ by

$$
\mathbb{O}_{(A, \phi)}=(\tilde{\nabla} s)_{(A, \phi)} \oplus \frac{1}{\sqrt{2} e} C_{(A, \phi)}^{\dagger}: T_{(A, \phi)}(\mathcal{A} \times \Gamma(E)) \longrightarrow \mathcal{W}_{(A, \phi)} \oplus \mathfrak{G},
$$

where we use the notation of Appendix A. At each vortex solution $(A, \phi) \in$ $\mathcal{V}$, identifying the target of $\mathbb{O}$ with the space

$$
\Omega^{0,1}\left(M ; \phi^{*} \text { Vert }\right) \oplus \Omega^{0,2}\left(M ;\left(\mathfrak{g}_{P}\right)_{\mathbb{C}}\right) \oplus \Omega_{+}^{0}\left(M ; \mathfrak{g}_{P}\right) \oplus \Omega^{0}\left(M ; \mathfrak{g}_{P}\right),
$$

a calculation shows that this operator can be written in local coordinates as

$$
\mathbb{O}_{(A, \phi)}=\left[\begin{array}{c}
\left\{\left[\left(\phi^{*} \nabla^{A}\right)^{0,1} \chi\right]_{\bar{\alpha}}^{j}+\psi_{\bar{\alpha}}^{a} \hat{e}_{a}^{j}\right\} \mathrm{d} \bar{z}^{\alpha} \otimes \hat{\phi}^{*}\left(\frac{\partial}{\partial w^{j}}\right) \\
\frac{1}{\sqrt{2} e}\left\{\overline{\left(D_{A} \psi\right)_{\alpha \beta}^{a}}-\overline{\left(D_{A} \psi\right)_{\beta \alpha}^{a}}\right\}\left(\mathrm{d} \bar{z}^{\alpha} \wedge \mathrm{d} \bar{z}^{\beta}\right) e_{a} \\
\frac{1}{\sqrt{2} e} \Im \mathfrak{m}\left[4 h^{\alpha \bar{\beta}}\left(D_{A} \psi\right)_{\alpha \bar{\beta}}^{a}+e^{2} \kappa^{a b} h_{j \bar{k}} \overline{\hat{e}_{b}^{k}} \chi^{j}\right] e_{a} \\
\frac{-1}{\sqrt{2} e} \Re \mathfrak{e}\left[4 h^{\alpha \bar{\beta}}\left(D_{A} \psi\right)_{\alpha \bar{\beta}}^{a}+e^{2} \kappa^{a b} h_{j \bar{k}} \overline{\hat{e}_{b}^{k}} \chi^{j}\right] e_{a}
\end{array}\right] .
$$

The first three components of $\mathbb{O}_{(A, \phi)}$ correspond to the operators obtained from the linearization of the three vortex equations at the point $(A, \phi) \in \mathcal{V}$. The last component of $\mathbb{O}_{(A, \phi)}$, roughly speaking, measures the orthogonality of a tangent vector in $T_{(A, \phi)}(\mathcal{A} \times \Gamma(E))$ to the $\mathcal{G}$-orbit of $(A, \phi)$. The cokernels of the operators $\mathbb{O}_{(A, \phi)}$ for all $(A, \phi)$ in $\mathcal{V}$ define a vector bundle cok $\mathbb{O} \rightarrow \mathcal{V}$. Taking the quotient by $\mathcal{G}$ one obtains another vector bundle, and the symbol $\mathcal{E}(\operatorname{cok} \mathbb{O} / \mathcal{G} \rightarrow \mathcal{V} / \mathcal{G})$ denotes the Euler class of this bundle. 
Finally, the symbol $w_{\theta}$ represents the Weil homomorphism (50). More precisely, what we mean in formula (68) is

$$
w_{\theta}[W(\alpha, \gamma)]:=\int_{\gamma} w_{\theta}\left[\mathcal{O}_{\alpha}\right]
$$

Thus we see that the correlation functions $Z$ can be computed by integrating certain closed differential forms over the moduli space $\mathcal{V} / \mathcal{G}$. Moreover, using the results of Section 6, we have that

$$
Z=\int_{\mathcal{V} / \mathcal{G}}\left[\prod_{i} \int_{\gamma_{i}} w_{\beta}\left(\hat{\Upsilon}^{*} \alpha_{i}\right)\right] \wedge \mathcal{E}(\operatorname{cok} \mathbb{O} / \mathcal{G} \rightarrow \mathcal{V} / \mathcal{G})
$$

Comparing this formula with the definition of the Hamiltonian GromovWitten invariants in [7], one recognizes that, in the case where $\operatorname{cok} \mathbb{O}=0$, our correlation functions essentially coincide with those invariants.

\subsection{The moduli space of vortex solutions}

As was seen above, the moduli space $\mathcal{V} / \mathcal{G}$ is of the utmost importance for the calculation of the correlation functions of our theory. In this subsection we will report some properties of this moduli space. The majority of the results here comes from references $[6,7,13]$. As in those references, we will restrict ourselves to the case where $M$ is a compact Riemann surface.

For the general gauged sigma-model the moduli space $\mathcal{V} / \mathcal{G}$ is not necessarily a smooth manifold. When it is a smooth manifold, or at least in each smooth region, the tangent space $T_{[A, \phi]} \mathcal{V} / \mathcal{G}$ can be identified with the kernel of the operator $\mathbb{O}_{(A, \phi)}$. In the light of the discussion below (70) this identification is very natural, since a tangent vector belongs to $\operatorname{ker} \mathbb{O}_{(A, \phi)}$ exactly if it satisfies the linearized vortex equations and is perpendicular to the $\mathcal{G}$-orbit of $(A, \phi)$. More generally, to decide whether $\mathcal{V} / \mathcal{G}$ is or is not smooth, one should study in detail the linearized equations and the orthogonality condition, i.e. the operator $\mathbb{O}$. In fact, the kernel, cokernel and index of $(\mathbb{O}$ are the relevant objects that characterize the local structure of $\mathcal{V} / \mathcal{G}$.

Following this cue, the first important result is the virtual dimension of the space $\mathcal{V} / \mathcal{G}$. This is given by the real index of the Fredholm operator $\mathbb{O}$ and coincides with the actual dimension of $\mathcal{V} / \mathcal{G}$ on smooth regions. Notice that, for $M$ a Riemann surface, the second component of this operator in (70) should be discarded, as the vortex equation $F_{A}^{0,2}=0$ is trivially satisfied. The computation of the index of $\mathbb{O}$ was performed in references $[6,13]$, and 
the result is

$$
\text { ind } \begin{aligned}
\mathbb{O}_{(A, \phi)} & =\operatorname{dim} \operatorname{ker} \mathbb{O}_{(A, \phi)}-\operatorname{dim} \operatorname{cok} \mathbb{O}_{(A, \phi)} \\
& =\left(\operatorname{dim}_{\mathbb{C}} X-\operatorname{dim} G\right)(2-2 g)+2\left\langle c_{1}^{G}(T X),[\phi]\right\rangle .
\end{aligned}
$$

In this formula $g$ is the genus of $M, c_{1}^{G}(T X)$ is the equivariant first Chern class of $T X$ - which belongs to $H_{G}^{2}(X ; \mathbb{Z})$ - and $[\phi]$ is the class in $H_{2}^{G}(X ; \mathbb{Z})$ determined by the section $\phi$. In practice we have that $\left\langle c_{1}^{G}(T X),[\phi]\right\rangle$ equals the first Chern number of the bundle $\phi^{*}$ Vert $\rightarrow M$.

Observe that different connected components of the moduli space $\mathcal{V} / \mathcal{G}$ may have different dimensions, depending on the class $[\phi]$. In fact, in formulas such as (67) and (68), one usually fixes a class $B \in H_{2}^{G}(M ; \mathbb{Z})$, and then only integrates over the fields $\phi$ such that $[\phi]=B$. In the case of the vortex solutions this defines a subset $\mathcal{V}_{B} \subset \mathcal{V}$ which can be shown to be $\mathcal{G}$-invariant.

Still regarding the smoothness of the moduli space, the best one can usually do is to guarantee this smoothness on the (typically open) subset $\mathcal{V}^{*} \subset \mathcal{V}$ of the so-called irreducible solutions. In fact, over the irreducible solutions, a sufficient condition for this smoothness is the vanishing of the cokernel of $\mathbb{O}_{(A, \phi)}$, or in other words the surjectivity of this operator. We will now give the definition of irreducible solution and state a condition that is equivalent to the surjectivity of $\mathbb{O}_{(A, \phi)}$. Both of these come from Reference [7].

Definition. A solution $(A, \phi)$ of the vortex equations is called irreducible if there exists a point $z \in M$ such that the stabilizer of $\hat{\phi}(z) \in X$ is trivial, and the intersection $\hat{\mathfrak{g}}_{\hat{\phi}(z)} \cap J_{X} \hat{\mathfrak{g}}_{\hat{\phi}(z)}$ is zero. (Here $\hat{\mathfrak{g}}_{\hat{\phi}(z)}$ denotes the image of the linear map $\mathfrak{g} \rightarrow T_{\hat{\phi}(z)} X$ associated to the $G$-action on $X$.) We note that the subset $\mathcal{V}^{*} \subset \mathcal{V}$ of irreducible vortex solutions is $\mathcal{G}$-invariant, and that $\mathcal{G}$ acts freely on it.

Proposition. Consider the operator

$$
\begin{aligned}
L_{(A, \phi)}: & \Omega^{0}\left(M ; \phi^{*} \text { Vert }\right) \oplus \Omega^{0,1}\left(M ;\left(\mathfrak{g}_{P}\right)_{\mathbb{C}}\right) \longrightarrow \Omega^{0,1}\left(M ; \phi^{*} \text { Vert }\right), \\
\left(V, \tau_{\bar{\alpha}}^{a}(z) \mathrm{d} \bar{z}^{\alpha} \otimes e_{a}\right) & \longmapsto\left(\phi^{*} \nabla^{A}\right)^{0,1} V+\tau_{\bar{\alpha}}^{a}(z) \mathrm{d} \bar{z}^{\alpha} \otimes\left(\hat{e}_{a}\right)_{\hat{\phi}(z)}
\end{aligned}
$$

where $\left(\phi^{*} \nabla^{A}\right)^{0,1}$ is as in (37). Then $\mathbb{O}_{(A, \phi)}$ is surjective if and only if both $C_{(A, \phi)}$ and the adjoint $L_{(A, \phi)}^{\dagger}$ are injective.

Remark. An immediate consequence of the results above is that if $L_{(A, \phi)}^{\dagger}$ is injective for all $(A, \phi) \in \mathcal{V}^{*}$, then the moduli space $\mathcal{V}^{*} / \mathcal{G}$ has a natural structure of smooth manifold. 
Remark. The statement of the proposition above is a bit stronger than proposition 4.8 (iii) of [7], but follows directly from the proof presented there. More specifically, refer back to that proof, call $L_{\phi}^{\mathbb{C}}$ the operator

$$
\Omega^{1}\left(M ;\left(\mathfrak{g}_{P}\right)_{\mathbb{C}}\right) \longrightarrow \Omega^{1}\left(M ; \phi^{*} \text { Vert }\right), \quad \tau \mapsto \tau_{\mu}^{a}(x) \mathrm{d} x^{\mu} \otimes\left(\hat{e}_{a}\right)_{\hat{\phi}(x)},
$$

and $L_{\phi}$ its restriction to $\Omega^{1}\left(M ; \mathfrak{g}_{P}\right)$. Then it is enough to notice that $2\left(L_{\phi} L_{\phi}^{\dagger} \eta\right)^{0,1}=L_{\phi}^{\mathbb{C}}\left(L_{\phi}^{\mathbb{C}}\right)^{\dagger} \eta$ for all $\eta$ in $\Omega^{0,1}\left(M ; \phi^{*}\right.$ Vert $)$. Moreover, $\left(L_{\phi}^{\mathbb{C}}\right)^{\dagger} \eta=0$ if and only if $L_{\phi}^{\dagger} \eta=L_{\phi}^{\dagger}\left(\eta \circ J_{M}\right)=0$.

One of the issues raised by the previous proposition is the injectivity of $C_{(A, \phi)}$, the operator in (12) that represents the infinitesimal gauge transformations. This issue is interesting for its own sake, and also came up in the discussion below (53). In fact, notice that $C_{(A, \phi)}$ is injective if and only if the $\mathcal{G}$-stabilizer of $(A, \phi)$ is discrete. Regarding these matters there exists the following result.

[7] Suppose that $M$ and $\mu^{-1}(0)$ are compact, and that 0 is a regular value of $\mu$ (resp. $G$ acts freely on $\left.\mu^{-1}(0)\right)$. Then there exists a constant $K>0$ such that, if $e^{2}(\operatorname{Vol} M) \geq K T_{[\phi]}$, every solution $\left(A, \phi^{\prime}\right)$ of the vortex equations with $\left[\phi^{\prime}\right]=[\phi]$ has a discrete (resp. trivial) $\mathcal{G}$-stabilizer.

Here $[\phi] \in H_{2}^{G}(M ; \mathbb{Z})$ is the equivariant homology class already mentioned above (which, by the way, is the same for homotopic sections $\phi$ ), and $T_{[\phi]}$ is the topological energy of (7). This result means that for large enough Riemann surfaces the group of gauge transformations acts (locally) freely on the space of vortex solutions. In the case of abelian actions we prove the following result in Appendix B.

Suppose that $G$ is a torus, that $M$ and $X$ are compact, and that the constant $(\operatorname{deg} P) /\left(e^{2} \operatorname{Vol} M\right)$ is a regular value of $\mu$ (see the appendix for the definition of $\operatorname{deg} P)$. Then every solution of the vortex equations has a discrete $\mathcal{G}$ stabilizer. If, furthermore, the torus action on $X$ has no non-trivial finite stabilizers, then the $\mathcal{G}$-action on the set of vortex solutions is free.

Remark. The last two propositions are true even if $\operatorname{dim}_{\mathbb{C}} M>1$. In the second one, the condition of compact $X$ can be very much weakened (see the remark in Appendix B); in particular the result is still valid for linear torus actions on $\mathbb{C}^{n}$.

Remark. Suppose that $G$ is a $n$-torus, that $X$ is compact of complex dimension $n$, and that the constant $(\operatorname{deg} P) /\left(e^{2} \operatorname{Vol} M\right)$ lies in the interior of the 
polytope $\mu(X)$. Then it is not difficult to show that the $\mathcal{G}$-action on $\mathcal{V}=\mathcal{V}^{*}$ is free and that the operators $L_{(A, \phi)}^{\dagger}$ are injective for every vortex solution $(A, \phi)$. (We omit the proof here.) According to the discussion above, this implies the smoothness of the moduli space $\mathcal{V} / \mathcal{G}$, in agreement with the results of $[2]$.

\subsection{About the invariants}

\subsubsection{Wall-crossing phenomena}

In this subsection we want to illustrate the so-called wall-crossing phenomena for the Hamiltonian Gromov-Witten invariants [6]. These refer to the occurrences where a finite deformation of the parameters of the topological Lagrangian leads to a change in the value of the invariants. This is in apparent contradiction with the argument evoked at the end of 7.1 about the vanishing of the vacuum expectation value of $Q$-exact operators. In fact, it will be very clear in the example below how this argument can indeed sometimes fail.

For our illustrative example we will consider the case of a toric action on $X=\mathbb{C P}^{n}$. Defining the constant

$$
\tau_{0}:=\frac{(\operatorname{deg} P)}{\left(e^{2} \operatorname{Vol} M\right)} \quad \in \mathfrak{g} \simeq \mathbb{R}^{n},
$$

it was shown in [2] that the moduli space of vortex solutions is a fixed nonempty manifold $\mathcal{V}$ whenever $\tau_{0}$ lies in the interior of the convex polytope $\mu(X)$. If $\tau_{0}$ lies outside this polytope the moduli space is empty.

Now suppose that we deform the moment map $\mu$ by adding to it a constant $\tau \in \mathbb{R}^{n}$. This corresponds to a deformation of the parameters of the topological Lagrangian, and so the heuristical arguments of Section 7.1 would seem to imply that the correlation functions are invariant by this deformation. In particular all the correlation functions should vanish, since for $\tau$ big enough $\tau_{0}$ lies outside $\mu(X)$, and so the integral in (68) is over the empty set. But in Reference [13] it was computed that for $X=\mathbb{C P}^{1}$ and $\tau_{0} \in \operatorname{int} \mu(X)$ there is a non-zero Hamiltonian Gromov-Witten invariant, so the heuristical argument must fail at some point. We will now see explicitly how this failure comes about. 
Take the gauge fermion $\Psi$ of (31) and substitute $\mu$ for $\mu+\epsilon$, where $\epsilon$ is a small constant in $\mathbb{R}^{n}$. Then the change in $Q \Psi$ is

$$
\delta Q \Psi= \pm \frac{i e}{\sqrt{2}}(C, \epsilon),
$$

and so the partition function, which is the simplest invariant, changes by

$$
\delta Z=\mp \int \mathcal{D} \text { (fields) } \frac{i e}{\sqrt{2}}(C, \epsilon) e^{-Q \Psi} .
$$

Using the explicit formula for $Q \Psi$ and integrating out the field $C$, the corresponding equation of motion is

$$
C=\mp \frac{i}{2 \sqrt{2} e t}\left(\Lambda F_{A}+e^{2} \mu \circ \phi\right),
$$

and so one recognizes that $\delta Z$ is just the vacuum expectation value of

$$
-\frac{1}{4 e t} \int_{M} \kappa_{a b}\left(\Lambda F_{A}+e^{2} \mu \circ \phi\right)^{a} \epsilon^{b}=\frac{e(\operatorname{Vol} M)}{4 t} \kappa(v, \epsilon),
$$

with

$$
v=\tau_{0}-\frac{1}{(\operatorname{Vol} M)} \int_{M} \mu \circ \phi .
$$

Now, if $\tau_{0}$ lies in the interior of $\mu(X)$, then the vector $v$ may have any orientation in $\mathbb{R}^{n}$ as $\phi$ varies, and so it is certainly possible that the expectation value of $\kappa(v, \epsilon)$ vanishes. However, when $\tau_{0}$ lies in the boundary or exterior of $\mu(X)$, the vector $v$ always lies in the same semi-space of $\mathbb{R}^{n}$, independently of $\phi$. Hence in this case the expectation value of $\kappa(v, \epsilon)$ cannot vanish for a generic infinitesimal deformation $\epsilon$, and so $\delta Z$ will not vanish.

We conclude that a finite deformation of $\mu$ may well leave the partition function $Z$ invariant, but only as long as $\tau_{0}$ remains in the interior of the polytope $\mu(X)$. When $\tau_{0}$ crosses the boundary of $\mu(X)$, a jump in the value of $Z$ is expected. This point of $\tau_{0}$ crossing the boundary of $\mu(X)$ corresponds as well to a drastic change in the ground states of the theory, as the moduli space of vortex solutions jumps from $\mathcal{V}$ to the empty set. All this is very similar to the runaway vacua phenomena in supersymmetric quantum mechanics [8, ch. 12.6].

The picture that seems to arise is that, in general, the space of parameters of the topological Lagrangian (and these include metrics, complex structures, ...) is divided into different regions by internal walls. A small deformation of the parameters will leave the correlation functions invariant, but when a wall is crossed the correlation functions may jump. 


\subsubsection{Adiabatic limit and Gromov-Witten invariants}

In this final paragraph we would like to mention another interesting property of the vortex equations, namely the correspondence between pseudoholomorphic curves in the symplectic quotient $X / / G$ and solutions of the vortex equations in the adiabatic limit $e \rightarrow \infty$. We assume here that $G$ acts freely in $\mu^{-1}(0)$, so that the symplectic quotient $X / / G:=\mu^{-1}(0) / G$ is in a natural way an almost Kähler manifold.

In the limit $e \rightarrow \infty$ the vortex equations (8) for $M$ a Riemann surface become

$$
\bar{\partial}^{A} \phi=0 ; \quad \mu \circ \phi=0
$$

It is not difficult to show that any solution of these equations descends to a pseudo-holomorphic map $\bar{\phi}: M \rightarrow X / / G$, and that gauge-equivalent solutions descend to the same map. Furthermore, any pseudo-holomorphic curve $\bar{\phi}$ lifts to a solution of (72) on the bundle $P=\bar{\phi}^{*}\left(\mu^{-1}(0) \rightarrow X / / G\right)$, and any two different lifts are gauge equivalent [9]. (In passing, the connection $A$ of the lift is the pull-back by $\bar{\phi}$ of the connection $\bar{A}$ on $\mu^{-1}(0) \rightarrow X / / G$ determined by the $G$-invariant metric on $\mu^{-1}(0)$.) One can therefore identify the moduli space of solutions of (72) with the space of pseudo-holomorphic curves on $X / / G$ such that $P \simeq \bar{\phi}^{*}\left(\mu^{-1}(0) \rightarrow X / / G\right)$.

On the other hand one would expect that for $e$ big enough there should be some sort of close correspondence betweeen the solutions of the vortex equations (8) and the solutions of (72). In particular the Hamiltonian Gromov-Witten invariants of $X$ - which study the moduli space of vortex solutions - should be able to tell something about the Gromov-Witten invariants of $X / / G$ - which study the space of pseudo-holomorphic curves. These matters were studied in detail in reference [9], and under suitable conditions on $M$ and $X$, one such relation was established. In the particular case where $X$ is a complex vector space acted by a torus and $X / / G$ is a toric variety, a very strong correspondence had been previously established in [12].

\section{Acknowledgments}

I would like to thank Prof. N. S. Manton for his encouragement, Professor Sir Michael Atiyah for a useful discussion, and Dr. David Tong for pointing out references [12,17]. I am supported by 'Fundação para a Ciência e Tecnologia', Portugal, through the research grant SFRH/BD/4828/2001. 


\section{Appendix A: The localization bundle}

In this appendix, we sketch how the gauged sigma-model can be fitted into the geometrical method for obtaining topological Lagrangians. We will have in mind the abstract description of this method given in [8, ch. 14], so only the features that are particular to our model will be described here.

One starts by considering the vector bundle over the space of fields $\mathcal{W} \rightarrow$ $\mathcal{A} \times \Gamma(E)$, whose fibre at a point $(A, \phi)$ of the base is

$$
\mathcal{W}_{(A, \phi)}=\Omega^{0,1}\left(M ; \phi^{*} \text { Vert }\right) \oplus \Omega_{+}^{0}\left(M ; \mathfrak{g}_{P}\right) \oplus \Omega^{0,2}\left(M ;\left(\mathfrak{g}_{P}\right)_{\mathbb{C}}\right)
$$

In this formula, $\Omega_{+}^{0}\left(M ; \mathfrak{g}_{P}\right)$ is the subspace of $\Omega^{0}\left(M ; \mathfrak{g}_{P}\right)$ defined by

$$
\Omega_{+}^{0}\left(M ; \mathfrak{g}_{P}\right):=\Lambda\left(\Omega^{2}\left(M ; \mathfrak{g}_{P}\right)\right)+\Omega^{0}\left(M ;\left(\mathfrak{g}_{0}\right)_{P}\right),
$$

where $\Lambda$ is the operator of $(9)$, and $\left(\mathfrak{g}_{0}\right)_{P}$ is the sub-bundle of $\mathfrak{g}_{P}$ constructed from the $\operatorname{Ad}_{G}$-invariant subspace

$$
\mathfrak{g}_{0}:=\operatorname{span}\{\mu(X)\} \subseteq \mathfrak{g} .
$$

We note in passing that, in the case where $X$ is a point and $\operatorname{dim}_{\mathbb{R}} M=4$, the space $\mathcal{W}_{(A, \phi)}$ defined above is isomorphic to the space of anti-self-dual $\mathfrak{g}_{P}$-valued forms on $M$. This is important because we want our construction to contain topological Yang-Mills theory as a special case.

The vector bundle $\mathcal{W}$ has a natural section defined by

$$
s(A, \phi)=\left(\bar{\partial}^{A} \phi, \frac{1}{\sqrt{2} e}\left(\Lambda F_{A}+e^{2} \mu \circ \phi\right), \frac{\sqrt{2}}{e} F_{A}^{0,2}\right) .
$$

Notice that the zero set of $s$ is the set of solutions of the vortex equations, and that the squared norm of $s$ is the non-topological term of the energy functional (6).

The group of gauge transformations $\mathcal{G}$ has a natural right action on the total space of the bundle $\mathcal{W}$ which lifts the usual $\mathcal{G}$-action on $\mathcal{A} \times \Gamma(E)$. The section $s$ is equivariant with respect to these actions, and so defines a section $\bar{s}$ of the quotient bundle

$$
\mathcal{W} / \mathcal{G} \longrightarrow(\mathcal{A} \times \Gamma(E)) / \mathcal{G} .
$$

This last bundle over the moduli space of fields is what is usually called the localization bundle. Although our Lagrangian and observables are ultimately meant to be defined on this bundle, it is easier to work "upstairs" on the bundle $\mathcal{W}$, and then include a "projection term" that brings all these quantities down to the quotient bundle (see [8]). 
In Section 3, the fields $A_{\alpha}^{a}(z)$ and $\hat{\phi}^{j}(z)$ were introduced as local coordinate functions on the space $\mathcal{A} \times \Gamma(E)$, which is the base of the bundle $\mathcal{W}$. Here we introduce the odd fields

$$
d_{\bar{\alpha}}^{j}(z), \quad c^{a}(z) \quad \text { and } \quad b_{\bar{\alpha} \bar{\beta}}^{a}(z)
$$

that should be regarded as odd coordinates on the fibre of $\mathcal{W}$ or to be more specific, respectively, on the spaces

$$
\Omega^{0,1}\left(M ; \phi^{*} \text { Vert }\right), \quad \Omega_{+}^{0}\left(M ; \mathfrak{g}_{P}\right) \quad \text { and } \quad \Omega^{0,2}\left(M ;\left(\mathfrak{g}_{P}\right)_{\mathbb{C}}\right) .
$$

So for example, if $\zeta=\zeta_{\bar{\alpha}}^{j} \mathrm{~d} \bar{z}^{\alpha} \otimes \hat{\phi}^{*}\left(\partial / \partial w^{j}\right)$ is an element of $\Omega^{0,1}\left(M ; \phi^{*}\right.$ Vert $)$, then the function $d_{\bar{\alpha}}^{j}(z)$ evaluated at $\zeta$ gives

$$
d_{\bar{\alpha}}^{j}(z)[\zeta]=\zeta_{\bar{\alpha}}^{j}(z)
$$

In Section 3, the operator $Q$ was defined as the differential of the $\mathcal{G}$ equivariant complex of $\mathcal{A} \times \Gamma(E)$. Here we extend that picture and define $Q$ to be the differential of the $\mathcal{G}$-equivariant complex of the total space of the bundle $\mathcal{W}$. When acting on functions, $Q$ obviously coincides with the exterior derivative $\tilde{\mathrm{d}}$ on $\mathcal{W}$, so

$$
\begin{aligned}
& Q b_{\bar{\alpha} \bar{\beta}}^{a}(z)=\tilde{\mathrm{d}}\left[b_{\bar{\alpha} \bar{\beta}}^{a}(z)\right]=: B_{\bar{\alpha} \bar{\beta}}^{a}(z) \\
& Q c^{a}(z)=\tilde{\mathrm{d}}\left[c^{a}(z)\right]=: C^{a}(z) \\
& Q d_{\bar{\alpha}}^{j}(z)=\tilde{\mathrm{d}}\left[d_{\bar{\alpha}}^{j}(z)\right]=: D_{\bar{\alpha}}^{j}(z)-\Gamma_{i k}^{j} \circ \hat{\phi}(z) \chi^{k}(z) d_{\bar{\alpha}}^{i}(z) .
\end{aligned}
$$

The rightmost equalities define the fields $B, C$ and $D$, which are odd 1forms on $\mathcal{W}$. Using the explicit (local) expression for the action of $\mathcal{G}$ on $\mathcal{W}$, and, as in Section 3, the definition of the differential $Q$ of the $\mathcal{G}$-equivariant complex, one can then compute what the action of $Q$ on $B, C$ and $D$ is. The result is given in the expressions (30).

The bundle $\mathcal{W} \rightarrow \mathcal{A} \times \Gamma(E)$ that we have been discussing has a natural connection $\tilde{\nabla}$. This connection is trivial on the sub-bundles corresponding to the last two summands of (A1), and, on the sub-bundle corresponding to the first summand, it is naturally induced by the Levi-Civita connection of $X$. More explicitly, let $S$ be any section of $\mathcal{W}$ and, according to (A1), decompose it as

$$
S=S_{1}+S_{2}+S_{3}
$$

where, locally,

$$
\begin{aligned}
S_{1}(A, \phi) & =\left[\left(S_{1}\right)_{\bar{\alpha}}^{j}(z)\right]_{(A, \phi)} \mathrm{d} \bar{z}^{\alpha} \otimes \hat{\phi}^{*}\left(\frac{\partial}{\partial w^{j}}\right) \\
S_{2}(A, \phi) & =\left[\left(S_{2}\right)^{a}(z)\right]_{(A, \phi)} e_{a} \\
S_{3}(A, \phi) & =\left[\left(S_{3}\right)_{\bar{\alpha} \bar{\beta}}^{a}(z)\right]_{(A, \phi)}\left(\mathrm{d} \bar{z}^{\alpha} \wedge \mathrm{d} \bar{z}^{\beta}\right) e_{a} .
\end{aligned}
$$


Then the connection $\tilde{\nabla}$ acts on each of these terms as

$$
\begin{aligned}
\tilde{\nabla} S_{1} & =\left\{\tilde{\mathrm{d}}\left[\left(S_{1}\right)_{\bar{\alpha}}^{j}(z)\right]+\Gamma_{k l}^{j} \circ \hat{\phi}(z) \chi^{l}(z)\left(S_{1}\right)_{\bar{\alpha}}^{k}(z)\right\} \mathrm{d} \bar{z}^{\alpha} \otimes \hat{\phi}^{*}\left(\frac{\partial}{\partial w^{j}}\right) \\
\tilde{\nabla} S_{2} & =\tilde{\mathrm{d}}\left[\left(S_{2}\right)^{a}(z)\right] e_{a} \\
\tilde{\nabla} S_{3} & =\tilde{\mathrm{d}}\left[\left(S_{3}\right)_{\bar{\alpha} \bar{\beta}}^{a}(z)\right]\left(\mathrm{d} \bar{z}^{\alpha} \wedge \mathrm{d} \bar{z}^{\beta}\right) e_{a} .
\end{aligned}
$$

Notice also that this can be rewritten as

$$
\tilde{\nabla} S=S^{*}\left[D_{\bar{\alpha}}^{j}\right] \mathrm{d} \bar{z}^{\alpha} \otimes \hat{\phi}^{*}\left(\frac{\partial}{\partial w^{j}}\right)+S^{*}\left[C^{a}\right] e_{a}+S^{*}\left[B_{\bar{\alpha} \bar{\beta}}^{a}\right]\left(\mathrm{d} \bar{z}^{\alpha} \wedge \mathrm{d} \bar{z}^{\beta}\right) e_{a} .
$$

As mentioned in Section 4, once the field content of the theory is established and the $Q$-action on the fields is known, there is a fairly standard procedure to construct a $Q$-exact Lagrangian for the topological theory. The abstract method is very well described in [8], and the necessary calculations are described in Section 4.

\section{Appendix B: A proof from Section 7}

In this appendix, we want to prove the third proposition of Section 7.2. The assumptions are that $G=T^{n}, M$ and $X$ are compact, and that the constant $(\operatorname{deg} P) /\left(e^{2} \operatorname{Vol} M\right)$ is a regular value of the moment map $\mu$. Here

$$
\operatorname{deg} P:=-\int_{M} \Lambda F_{A} \quad \in \mathfrak{g} \simeq \mathbb{R}^{n},
$$

and it is not difficult to check that this constant does not depend on the connection $A$.

For the first part of the proposition, it is enough to show that for every vortex solution $(A, \phi)$ the operator $C_{(A, \phi)}=C_{A}+C_{\phi}$ of (52) is injective. So let $\varepsilon \in \mathfrak{G} \simeq C^{\infty}\left(M ; \mathbb{R}^{n}\right)$ be an infinitesimal gauge transformation such that

$$
\begin{aligned}
& C_{A}(\varepsilon)=D_{A} \varepsilon=\mathrm{d} \varepsilon=0 ; \\
& C_{\phi}(\varepsilon)=-\varepsilon^{a}(x) \hat{\phi}^{*}\left(\hat{e}_{a}\right)=0 .
\end{aligned}
$$

The first equation tells us that $\varepsilon$ is constant. Calling $\theta \in \mathbb{R}^{n}$ the constant value of $\varepsilon$, the second equation tells us that $\left.\hat{\theta}\right|_{\hat{\phi}(x)}=0$, where $\hat{\theta}$ is the vector field on $X$ induced by $\theta$. Using the definition of moment map, this means that $\hat{\phi}(x) \in X$ is a critical point of the function $H_{\theta}:=\langle\mu, \theta\rangle$ for all $x \in M$. Now denote by $B_{\theta}^{r}$ the connected components of the critical set $\operatorname{Crit}\left(H_{\theta}\right) \subset$ $X$. These components are preserved by the torus action, since $\mu$ is $T^{n_{-}}$ invariant and the orbits of the action are connected. One can therefore 
define the associated bundles

$$
E_{\theta}^{r}:=P \times_{T^{n}} B_{\theta}^{r}
$$

which are connected subsets of $E=P \times_{T^{n}} X$. Since $\phi(M) \subset E$ is connected, the discussion above implies that $\phi(M)$ is contained in one of the $E_{\theta}^{r}$ 's, say $E_{\theta}^{0}$. Thus

$$
\mu \circ \phi(M) \subset \mu\left(E_{\theta}^{0}\right)=\mu\left(B_{\theta}^{0}\right) .
$$

Now by the lemma below, $\mu\left(B_{\theta}^{0}\right)$ is a convex polytope in $\mathbb{R}^{n}$, and so the constant

$$
\frac{\operatorname{deg} P}{e^{2} \operatorname{Vol} M}=\frac{-1}{e^{2} \operatorname{Vol} M} \int_{m} \Lambda F_{A}=\frac{1}{\operatorname{Vol} M} \int_{M} \mu \circ \phi
$$

certainly belongs to this polytope. This finally shows that, unless $\theta=0$, the constant $(\operatorname{deg} P) /\left(e^{2} \operatorname{Vol} M\right)$ is a critical value of $\mu$, and the proof of the first part is complete.

For the second part of the proposition, assume furthermore that all the $G$-stabilizers in $X$ are either trivial or a subtorus of $T^{n}$. To obtain at the end a contradiction, suppose that there existed a non-trivial gauge transformation $g \in C^{\infty}\left(M ; T^{n}\right)$ that preserved the pair $(A, \phi)$. The condition that $g$ preserves the connection $A$ implies that $g$ is a constant map, as is well known, and we call its image also $g \in G \backslash\{$ id $\}$. The condition $g(\phi)=\phi$, on the other hand, implies that

$$
\phi(M) \subset P \times_{T^{n}} \operatorname{Fix}\left(\rho_{g}\right) \subset E,
$$

where $\operatorname{Fix}\left(\rho_{g}\right) \subset X$ is the set of fixed points of the map $\rho_{g}$, and so is $T^{n}$ invariant. But the initial assumption on the $G$-stabilizers in $X$ then implies that each point of $\operatorname{Fix}\left(\rho_{g}\right)$ is preserved by a full subtorus of $T^{n}$, and in particular is a critical point of $\mu$. Thus

$$
\phi(M) \subset P \times_{T^{n}} \operatorname{Crit}(\mu) \subset E .
$$

Now, the proofs of Theorem 5.47 and Lemma 5.53 in [11] show that

$$
\operatorname{Crit}(\mu)=\bigcup_{\theta \in \mathbb{Z}^{n} \backslash\{0\}} \operatorname{Crit}\left(H_{\theta}\right)
$$

and that each $\operatorname{Crit}\left(H_{\theta}\right)$ - the critical set of $H_{\theta}$ - is a proper complex submanifold of $X$. Thus defining $E_{\theta}:=P \times_{T^{n}} \operatorname{Crit}\left(H_{\theta}\right)$, we have that

$$
\phi(M) \subset \bigcup_{\theta \in \mathbb{Z}^{n} \backslash\{0\}} E_{\theta},
$$

and that each $E_{\theta}$ is a proper complex submanifold of $E$ equipped with the integrable complex structure induced by $A$ (see Section 2.2 in [2]). On the other hand, since $\bar{\partial}^{A} \phi=0$, also $\phi(M)$ is a complex submanifold of $E$ (see [13]). This implies that the intersections $\phi(M) \cap E_{\theta}$ are analytic subvarieties of $\phi(M)$, and so it follows from (B1) and Baire's category theorem 
that there exists at least one $\theta \neq 0$ such that $\phi(M) \subset E_{\theta}$. Finally, arguing just as at the end of the proof of the first part, one concludes that the constant $(\operatorname{deg} P) /\left(e^{2} \operatorname{Vol} M\right)$ must be a critical value of $\mu$, which contradicts the assumptions.

Lemma. The image $\mu\left(B_{\theta}^{0}\right)$ is a convex polytope in $\mathbb{R}^{n}$.

Proof. Along the proof above we saw that $\operatorname{Crit}\left(H_{\theta}\right)$ and $B_{\theta}^{0}$ are compact Kähler submanifolds of $X$ that are preserved by the $T^{n}$-action. Thus the restriction of $\mu$ to $B_{\theta}^{0}$ is a moment map for the $T^{n}$-action on $B_{\theta}^{0}$. The lemma then follows directly from the well known convexity theorem.

Remark. Inspecting the proof of the proposition presented here, it is clear that the assumption of compact $X$ is only needed to guarantee the validity of the lemma above. Thus as long as the images by $\mu$ of the connected components of the critical sets $\operatorname{Crit}\left(H_{\theta}\right)$ are convex sets, the proposition is still valid, even if $X$ is not compact. This happens for instance with the linear torus actions on $\mathbb{C}^{n}$.

\section{References}

[1] M.F. Atiyah and L. Jeffrey, Topological Lagrangians and cohomology, J. Geom. Phys. 7 (1990), 119.

[2] J.M. Baptista, Vortex Equations in Abelian Gauged Sigma-Models, Commun. Math. Phys. 261 (2006), 161.

[3] N. Berline, E. Getzler and M. Vergne, Heat Kernels and Dirac operators, Springer-Verlag, Berlin, 1992.

[4] D. Birmingham, M. Blau, M. Rakowski and G. Thompson, Topological field theory, Phys. Rep. 209 (1991), 129.

[5] M. Blau, The Mathai-Quillen formalism and topological field theory, J. Geom. Phys. 11 (1993), 95.

[6] K. Cieliebak, A.R. Gaio and D. Salamon, J-Holomorphic curves, moment maps, and invariants of hamiltonian group actions, Int. Math. Res. Notices 16 (2000), 831.

[7] K. Cieliebak, R.A. Gaio, I. Mundet i Riera and D.A Salamon, The symplectic vortex equations and invariants of Hamiltonian group actions, J. Symplectic Geom. 1 (2002), 543.

[8] S. Cordes, G. Moore and S. Ramgoolam, Lectures on 2D Yang-Mills Theory, Equivariant Cohomology and Topological Field Theories, hepth/9411210. 
[9] R. Gaio and D. Salamon, Gromov-Witten Invariants of Symplectic Quotients and Adiabatic Limits, math.SG/0106157.

[10] V. Guillemin and S. Sternberg, Supersymmetry and Equivariant de Rham Theory, Springer-Verlag, Berlin, 1999.

[11] D. McDuff and D. Salamon, Introduction to symplectic topology, 2nd edition, Oxford University Press, New York, 1998.

[12] D. Morrison and M. Plesser, Summing the instantons: quantum cohomology and mirror symmetry in toric varieties, Nuclear Phys. B 440 (1995), 279.

[13] I. Mundet i Riera, Hamiltonian Gromov-Witten invariants, Topology 42 (2003), 525;

I. Mundet i Riera, Yang-Mills-Higgs Theory for Symplectic Fibrations, Ph.D. Thesis, UAM (Madrid), April 1999, math.SG/9912150.

[14] I. Mundet i Riera and G. Tian, A Compactification of the Moduli Space of Twisted Holomorphic Maps, math.SG/0404407.

[15] E. Witten, Topological quantum field theory, Comm. Math. Phys. 117 (1988), 353.

[16] E. Witten, Topological sigma models, Comm. Math. Phys. 118 (1988), 411.

[17] E. Witten, Phases of $N=2$ theories in two dimensions, Nucl. Phys. B403 (1993), 159. 
